\title{
"Satire Junge SATIREEEE": Nachrichtenparodien in Memes und deren Aushandlung im Kontext des Web 2.0.
}

\author{
Fabia Hultin Morger(Stockholm)
}

\begin{abstract}
Satire has been present in various different media throughout the centuries. With the rise of television, satire has made its way onto TV screens via various outlets including news parodies. As these TV shows began using social media, new forms of satire have appeared, among them satirical Internet Memes commenting on political events. The objects of interest in this study are Memes published by two German news parodies Heute Show and Extra 3 on the platform Facebook that thematise the G-20 summit, which took place in Hamburg in 2017. My data set consists of 27 Memes from the platforms Facebook, Instagram and Twitter, as well as the public Facebook comments published alongside these Memes. Using an empirical, data-driven approach to my investigation, I broach questions regarding the way Memes make use of satire and how they interact with the Internet as a medium, and in particular, their affordances on the platform Facebook.
\end{abstract}

\section{$1 \quad$ Einleitung}

Beschrieben als „Angriffsliteratur mit einem Spektrum vom scherzhaften Spott bis zur pathetischen Schärfe“ (Müller et al. 2007, s. v. Satire), ist Satire ein Genre, welches sich über Jahrhunderte stets den Medien und gesellschaftlichen Gegebenheiten der Zeit angepasst hat. Es mag daher nicht erstaunen, dass Satire auch im heutigen ,age of the Internet, mass media, and punk rock" (Caufield 2012: 5) weiterhin in verschiedenen Medien Verbreitung findet. Dies zeigt sich beispielsweise in satirischen Fernsehsendungen, sogenannten Nachrichtenparodien, welche eine Form der Übertragung des Genres Satire ins Medium Fernsehen darstellen. Von diesen Fernsehsendungen geschieht auch der Sprung in die sozialen Netzwerkplattformen wie Facebook, Instagram oder Twitter. Die vormals nur im Fernsehen im Halbstundenformat vorkommenden Nachrichtenparodien haben mittlerweile damit begonnen, auch die sozialen Netzwerke zur Verbreitung ihrer Satire zu nutzen. Getan wird dies, nebst dem Teilen von Filmclips oder Zitaten aus der Sendung, auch mit anderen, mehr an das Medium Internet angepassten Textsorten. Für diese Fallstudie von besonderer Relevanz sind Internet Memes, medienspezifische Sprache-Bild-Texte, welche von den beiden deutschen Satiresendungen Heute Show und Extra 3 auf ihren sozialen Netzwerken häufig verbreitet werden.

Die thematische Umrahmung für diese Fallstudie bildet der G-20 Gipfel in Hamburg. Dieser fand am 07.07. und 08.07.2017 statt und es trafen sich dort die 20 weltweit größten Wirtschaftsmächte zu Diskussionen über Wirtschaft und Politik. Der Gipfel markierte Donald 
Trumps ersten Besuch als US-Präsident in Deutschland. Die Wochen zuvor und danach waren, ebenso wie diese beiden Tage, von zahlreichen Demonstrationen geprägt, welche teilweise in Vandalismus ausarteten und mit Polizeigewalt unterdrückt wurden. Dementsprechend führte das Ereignis zu großen Debatten. Auch lange Zeit nach dem Gipfel wird dieser sporadisch immer noch thematisiert. Im Rahmen der hier vorliegenden Studie werden insgesamt 27 Memes, welche mit dem G-20-Gipfel thematisch verbunden sind, analysiert. Diese wurden von der Heute Show und Extra 3 auf den sozialen Netzwerkplattformen Facebook, Instagram und Twitter publiziert. Neben den Internet-Memes sind für diese Studie auch die zur Plattform Facebook gehörigen Kommentarspalten relevant für die Untersuchung und werden in die Analyse miteingenommen. Anhand dieses zweiteiligen, in sich geschlossenen Datensatzes wird untersucht, wie sich die Phänomene Satire und Meme einander anpassen und inwiefern die Affordanzen des Mediums Internet dazu beitragen, Satire in Form von Memes auszudrücken.

Ziel der Studie ist, anhand eines Fallbeispiels empirisch darzulegen, wie satirische Texte in Form von Memes innerhalb der sozialen Netzwerke ausgestaltet und rezipiert werden. Dabei wird auch berücksichtigt, welche Affordanzen des Mediums von den Satireproduzentinnen und -rezipienten ${ }^{1}$ genutzt werden und in welcher Form. Darüber hinaus soll gezeigt werden, ob und inwiefern die Memes und die dazugehörigen Kommentare zur Verhandlung der Machtpositionen im Diskurs angewandt werden.

Dementsprechend sollen folgende Fragestellungen zur Erfassung wesentlicher Aspekte des Gebrauchs von Memes innerhalb des genannten Rahmens beitragen:

- Wie werden die gesammelten Memes von deren Produzenten (also Extra 3 und Heute Show) als satirische Texte ausgestaltet?

- Wie tragen die Memes zur Verhandlung der Machtpositionen im Diskurs um den G-20 bei?

- Inwiefern spielen die Affordanzen des Webs 2.0 bei der Rezeption und Ausgestaltung der Memes eine Rolle?

Die hier vorliegende Studie versteht sich als eine erste, breit gehaltene Bestandsaufnahme eines komplexen und noch grösstenteils unerforschten Phänomens. Der Fokus ist darauf gerichtet, satirische Internet Memes in einem bestimmten medialen Kontext zu sichten und Vorarbeit für Analysen mit tiefergehenden Fragestellungen zu leisten.

\subsection{Was sind Memes?}

Theoretische Untersuchungen zum Phänomen Internet Memes haben in den letzten Jahren an Aufwind gewonnen. Damit einher geht eine anhaltende Diskussion darüber, wie ein Meme definiert werden kann. In der germanistischen Linguistik gehen die meisten Wissenschaftler von Osterroths Definition aus, nach der ein Meme ,grundsätzlich auf einem Bild, welches der Popkultur, der Politik oder dem Alltag entstammt und für die Meme-Verwendung rekontextualisiert [wird]" basiert (Osterroth 2015: 28). Osterroth bezeichnet Memes als Sprache-Bild-

\footnotetext{
${ }^{1}$ In der folgenden Studie werde ich bei den Personenbezeichnungen zwischen der femininen und maskulinen Form willkürlich wechseln.
} 
Texte (ibd.), eine Bezeichnung, welche auch die Memes in dieser Fallstudie beschreibt. Gemeint sind damit in erster Linie Image Macros, eine Subform der Memes, auf welche sich die Aufmerksamkeit in diesem Forschungsbeitrag fokussiert. Image Macros sind nach folgendem Schema aufgebaut:

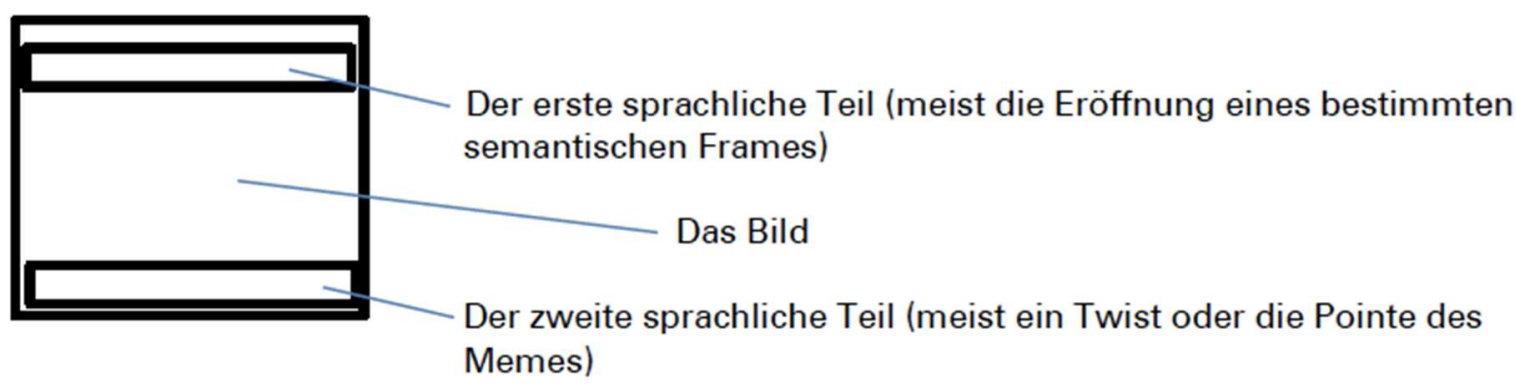

Abbildung 1: Aufbau eines Memes nach Osterroth 2015: 31 (Bülow/Merten/Johann 2018: 2).

Dies kann in der Praxis wie folgt aussehen:

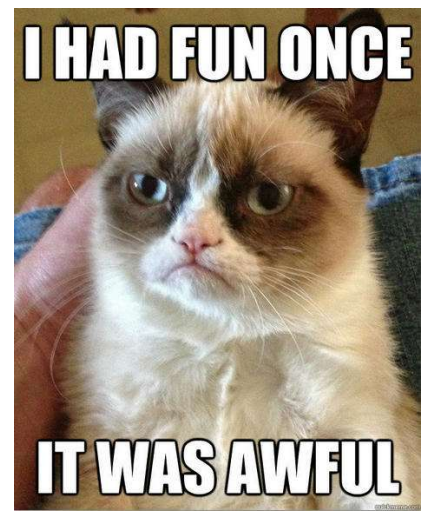

Abbildung 2: Populäres Grumpy Cat-Meme

Bülow/Merten/Johann erklären Osterroths Schema folgendermassen:

Das Setup befindet sich meistens im oberen Bereich des Bildes und dient vielfach der Eröffnung eines „bestimmten semantischen Frames“ [...]. Die Punchline ist im unteren Bereich des Bildes positioniert und konstruiert in der Regel die Pointe [...]

Bülow/Merten/Johann (2018: 2)

Die im Schema angegebene Struktur ist aber keineswegs obligatorisch und Abweichungen können durchaus vorkommen (ibd.), auch in den hier untersuchten Memes. Neben dem vor allem für Image Macros typischen Bild-Text-Format, ist auch die virale Verbreitung von Memes für deren Definition relevant, da wiederholtes Teilen auf dem Internet ein konstitutives Merkmal von Internet Memes darstellt (z. B. Castaño/Mauricio 2013, Davison 2012). Die in Memes vorhandenen Inhalte sind zumeist humorvoll. Osterroth schreibt Internet Memes eine ,inhärente Scherzhaftigkeit“ (Osterroth 2019: 53) zu und bezeichnet sie als ,pragmatische Nachfolger" (ibd.) von Emoticons, da Nachrichten durch sie als humorvoll markiert werden. Diese Humorhaftigkeit hindert jedoch Memes in keiner Weise daran, auch in politischen Diskursen ernsthafte Beiträge zu leisten: ,regardless of their emotional keying, political Memes are about making a point - participating in a normative debate [...]" (Shifman 2014: 120). 
Diese gegenwärtige sprachwissenschaftliche Konzeptualisierung von Internet Memes hat wenig gemein mit dem ursprünglichen Konzept, welches Dawkins 1976 beschrieb, als er den Begriff Meme prägte. Ursprünglich beschrieben Memes „units of cultural transmission“ (Dawkins 1976: 83), also kleinstmögliche Einheiten, welche, in Äquivalenz mit dem biologischen Gen, kulturelle Inhalte weiterverbreiten. Die Abgrenzung zwischen Dawkins' MemeKonzept und dem Internet Meme, kann, laut Osterroth (2019: 41f.), im deutschsprachigen Raum durch die Abgrenzung von der deutschen Übersetzung des Dawkins'schen Konzepts Mem (wobei in der deutschen Schreibweise das $e$ ausgelassen wird) und dem Anglizismus Meme, welcher die heutigen Internet Memes beschreibt, geschehen. Für die hier vorliegende Studie wird, in Anlehnung an Osterroths Definition der Anglizismus Meme verwendet, um Internet Memes wie etwa Image Macros zu beschreiben.

\subsection{Memes interpretieren und verstehen}

Die Inhalte von Memes sind eng mit dem Begriff der Ironie verbunden. So kategorisiert Osterroth (2019: 56) Memes als „,ironische, expressive Sprechakte“. Laut Schwarz-Friesel (2009: 223) ist die Haupteigenschaft von Ironie „dass das Gegenteil von dem, was gemeint ist, gesagt wird und dass eine (in der Regel) negative Bewertung des Sprechers übermittelt wird“. Von anderen Formen der Unehrlichkeit unterscheidet sich die Ironie dadurch, dass durch sie einerseits Einstellungen und Gefühle ausgedrückt werden, die eigentlich nicht existieren, andererseits aber auch zu verstehen gegeben werden muss, dass man diese Einstellungen und Gefühle eigentlich nicht besitzt (Lapp 1992: 141). Der Rezipient der Ironie muss sich der Ironie bewusst sein und darf die ironische Äusserung nicht als aufrichtig wahrnehmen. Hierfür ist die von Schwarz-Friesel thematisierte „Fähigkeit, die emotiven (Beziehungs)Aspekte von Gesprächen zu erkennen und zu würdigen“ (Schwarz-Friesel 2009: 230), von Bedeutung. Das Verständnis des ironischen Sprechakts hängt von der emotionalen Intelligenz der Leserin ab (cf. ibd.: 224). Für die korrekte Interpretation eines Memes wird dementsprechend ein Rezipient benötigt, welcher in der Lage ist, diese ironische Implikatur zu entschlüsseln.

Die korrekte Interpretation von Memes beruht zumeist auf einem kontextuellen Vorwissen. Memes können nicht von allen Lesern gleichermaßen verstanden werden und sind teilweise auch bewusst als Insiderwitz angelegt. Grundlingh (2018: 159) beschreibt diese Notwendigkeit wie folgt: „for a Meme to be a successful illocutionary act, the context of the Meme (the text and the image) must be interpreted within the context of the larger communication“. Memes sind also stets in einen größeren Diskurs eingebunden, innerhalb dessen sie als ironische Texte funktionieren.

\subsection{Satire: Eine Begriffsdiskussion}

Der titelbildende Ausspruch „Satire Junge SATIREEEE“ zeigt bereits die Wichtigkeit des Satirebegriffs für diese Studie auf. Es handelt sich bei diesem Zitat um einen Ausschnitt aus der gesammelten Kommentarsektion, wo der satirische Inhalt des dazugehörigen Memes diskutiert und die Definition von Satire verhandelt wird. Die Definitionsversuche in den Kommentarspalten spiegeln die Definitionsversuche im wissenschaftlichen Diskurs wider. So beschreibt Brummack den Satirebegriff als einen Begriff ,,von irritierender Vieldeutigkeit“ 
(Brummack 1971: 275). Brummack (ibd.: 282) definiert Satire als ,ästhetisch sozialisierte Aggression“, wobei eine psychologische, eine soziale und eine ästhetische Dimension allesamt konstitutive Elemente des Satirebegriffs sind. Die psychologische Dimension stellt eine „am Text ablesbare Aggression dar“ (ibd.). Dies geschieht in einem sozialisierten Rahmen: Die Produzenten der Memes nehmen gegenüber ihren Leserinnen Stellung und wollen ein gewisses Normsystem vermitteln. Die ästhetische Dimension sieht Brummack ,in der Besonderheit der beiden ersten [Dimensionen, also der psychologischen und sozialen] bedingt“ (ibd.). Texte wie die Internet Memes, welche in einem sozialen Rahmen eine am Text ablesbare Kritik ausüben, sind demnach zwangsläufig auch ästhetisch. Nach Brummacks Festlegung des Satirebegriffs können also Memes durchaus zu Satire zählen. Alle Dimensionen lassen sich auch in den hier erforschten Memes finden.

Eine tiefgreifende Diskussion des Satirebegriffs ist aber nicht Ziel dieser Studie. Die Einstufung der im Datenset gesammelten Internet Memes als Satire bedient sich vor allen Dingen des Konzepts der Ethnokategorisierung und versteht Satire als Ethnokategorie. Ethnokategorien werden von Luginbühl/Perrin (2011: 583) als „die Kategorien, mit denen die Mitglieder einer Sprachgemeinschaft ihre Textsorten erfassen" definiert. Ethnokategorien spielen in dieser Analyse im Bezug auf die Satirebezeichnung eine wichtige Rolle. Die auf den beiden Facebookseiten publizierten Memes werden vielmehr deshalb als Satire identifiziert, weil die Betreiber der Seiten sowie die Kommentierenden diese als solche benennen. Am deutlichsten dargestellt wird dies in den „About"“-Sektionen der beiden Facebook-Seiten, welche eine Art Impressum darstellt. Bei der Heute Show wird auf Facebook folgende Information zur Sendung gegeben: „Heute Show - die preisgekrönte ZDF-Nachrichtensatire mit Oliver Welke“ (ZDF Heute Show auf Facebook: Intro). Bei Extra 3 zeigt sich ein ähnliches Bild. Auf der Homepage der Show auf den Seiten des NDR wird sie als „Satiremagazin“ charakterisiert (NDR-Homepage: Extra 3). Diese eindeutige Deklarierung des Inhalts als Satire hat vermutlich auch juristische Gründe. Dadurch, dass der Inhalt klar als satirisch angegeben ist, schützen sich die Seiten vor möglichen Verleumdungs- oder Ehrverletzungsklagen, da sie sich im Falle eines Rechtsstreits stets auf den Satirestatus des Seiteninhalts beziehen können. Die Einordnung der auf den Facebookseiten publizierten Internet Memes als Satire kann daher nur schon aus diesem Grund als gegeben angesehen werden.

\section{$2 \quad$ Material und Methode}

Die beiden für diese Studie ausgewählten Sendungen Extra 3 und Heute Show sind sich in ihren Formaten relativ ähnlich: Beide werden einmal die Woche auf öffentlich-rechtlichen Fernsehsendern (NDR und ZDF) ausgestrahlt und dauern etwa eine halbe Stunde. Während der Sendung kommentieren die jeweiligen Moderatoren (Oliver Welke bei der Heute Show und Christian Ehring bei Extra 3) das Geschehen in der deutschen Politiklandschaft und der Welt im Allgemeinen, wobei auch verschiedene Gastkommentatorinnen erscheinen. Optisch ähneln beide Sendungen dem Format der Nachrichtensendung, das live anwesende (lachende und applaudierende) Publikum stellt jedoch einen entscheidenden Unterschied dar (Genaueres zur Kategorisierung des Sendeformats im Abschnitt 3.2). Im Rahmen dieser Studie befasse ich mich mit den Memes, welche sich mit dem im Juli 2017 stattgefundenen G-20 Gipfel auseinandersetzen. Während des Ablaufs des G-20 Gipfels hielten beide Sendungen Sommerpau- 
se, sodass die gesammelten Memes in den meisten Fällen keinen Bezug auf eine entsprechende Sendung nehmen, sondern vielmehr als mediale Erweiterung zum Zuge kommen.

Gegenstand der Studie sind 27 Memes sowie die in den Facebook-Kommentarfeldern stattfindenden Interaktionen. Während bei der Auswahl der Memes auf alle sozialen Netzwerkplattformen zurückgegriffen wird, beschränkt sich die Analyse der Kommentare auf die Plattform Facebook. Dies hat folgende Gründe: Einerseits herrscht auf Facebook eine starke Partizipation der Userinnen, da Facebook für beide Sendungen eine der beliebtesten Plattformen ist. $^{2}$ Andererseits provozieren die Plattformen Twitter und Instagram frappierend unterschiedliche Kommentare, was sich, zumindest teilweise, durch die verschiedenen Affordanzen der Plattformen erklären lässt. Eine umfassende Untersuchung der Kommentare aller Plattformen würde eine größere, kontrastive Analyse benötigen, welche über den Rahmen dieser Studie hinausginge. Daher beschränke ich mich in dieser Analyse auf die Kommentare von Facebook. Von den gesammelten Memes ist eines nicht auf Facebook sondern nur auf den Plattformen Twitter und Instagram geteilt worden, was bedeutet, dass zu einem der Memes keine dazugehörigen Kommentare gesammelt werden konnten.

Im Fokus der Analyse liegt die Anwendung der Plattform aus Benutzerperspektive: Das gesammelte Datenset besteht daher aus den für die Benutzer sichtbaren Kommentare und Internet Memes, sowie Likes und Reaktionen darauf. Gesammelt wurden diese Daten durch Screenshots des Kommentarfeldes. Dennoch wurden die meisten Topkommentare am Tag der Publikation des Memes verfasst, was darauf hinweist, dass die Kommentarsektionen in den Tagen nach der Publikation des Memes am aktivsten sind und danach zumeist stagnieren. Später erscheinende Änderungen in den Kommentarspalten geschehen höchstens dann, wenn eine Nutzerin ihr Facebookprofil löscht, was die Kommentare verschwinden lässt. Der Fokus liegt hierbei bei der von den Sendungen veröffentlichten Bildbeschreibung, den Likes, den Shares, sowie den Topkommentaren und deren Rückantworten. Die Namen der Nutzerprofile werden jeweils ausgeblendet und kodifiziert, sodass immer noch ersichtlich ist, welche Nutzer in welchen Interaktionen teilnehmen. Ein Screenshot von der Kommentarsektion aus meinem Datenset sieht folgendermassen aus:

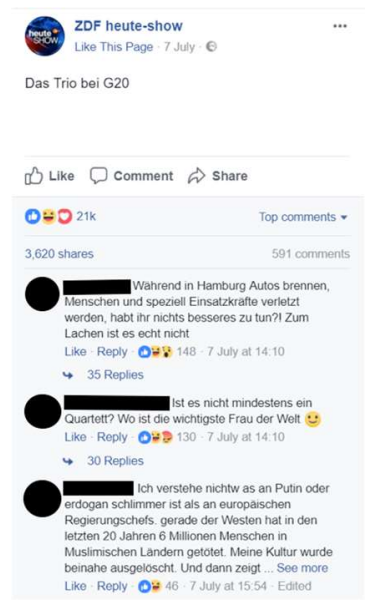

Abbildung 3: Überblick über eine Kommentarsektion bei Drei Plagezeichen-Meme

\footnotetext{
${ }^{2}$ Heute Show hat auf Twitter gerundet 880'000 Followers, auf Instagram 1.3 Millionen und auf Facebook 1,3 Millionen. Extra 3 hat auf Twitter 857'000 Followers, auf Instagram 559'000 und auf Facebook 1 Million. [24.03.2019]
} 
Eingesammelt werden die Topkommentare, also die sechs Kommentare, welche zuoberst in der Sektion angezeigt werden, da sie am meisten Likes und/oder Rückantworten haben, sowie Rückantworten auf die Topkommentare. Das Datenset besteht aus zusammengerechnet 2514 Kommentaren und Rückantworten.

Bei der Analyse handelt es sich um eine empirische Analyse, in welcher qualitative Ansätze im Vordergrund stehen. Eine Repräsentativität der Resultate wird nicht angestrebt. Dennoch sind quantitative Aussagen bezüglich der Inhalte der Kommentare von Relevanz, um einen tieferen Einblick ins Datenset zu gewähren.

\subsection{Ethische Überlegungen}

Ethische Überlegungen zur Studie beziehen sich vor allen Dingen auf die vorhandenen Kommentare. So sind die Identitäten der Internetnutzer zwar nicht mehr erkennbar, aber gleichzeitig sind die untersuchten Memes und deren Kommentarsektionen nach wie vor online auffindbar, und die Möglichkeit, dass die Lesenden dieser Studie diese finden, besteht. Schweden, wo die Forschung stattfindet und publiziert wird, steht, wie sämtliche EU-Länder, im Geltungsbereich der Datenschutz-Grundverordnung. Dies bedeutet, dass personenbezogene Daten auch in der Wissenschaft nur unter gewissen Bedingungen ohne Einwilligung der Kommentierenden gespeichert werden dürfen.

Bei den Kommentierenden eine Erlaubnis zur Verwendung für Forschungszwecke anzufordern wäre angesichts der Anzahl Kommentare allerdings nicht zweckmässig. Es besteht daher das Risiko, dass sich Kommentierende durch die Anwendung ihrer Kommentare zu Forschungszwecken in ihrer Integrität gekränkt fühlen. Zu bedenken ist aber, dass Facebookseiten wie die der Heute Show und Extra 3 öffentliche Plattformen sind, welche den Austausch mit der nicht-professionellen Öffentlichkeit suchen und fördern. Die auf den Seiten geposteten Kommentare werden folglich im Bewusstsein einer Veröffentlichung gepostet und provozieren zumeist auch eine Reihe von Rückantworten. Des Weiteren sieht die DatenschutzGrundverordnung Ausnahmen für die Verwendung von Daten für Forschungszwecke vor. In einem Beschluss der Schwedischen Ethikkommission (Etikprövningsnämden) wurde daher festgelegt, dass die ethischen Risiken der Studie aus den genannten Gründen zu verantworten seien.

\section{$3 \quad$ Mediale und diskursive Rahmung der untersuchten Memes}

\subsection{Web 2.0, Partizipation und ein neues Publikum}

Internet Memes sind eng verknüpft mit dem Medium, in dessen Rahmen sie publiziert und verbreitet werden, nämlich dem Internet. Innerhalb der Technologie des Internets lassen sich Memes als Produkt des Webs 2.0 zuordnen, welches ein ,zentraler Teil des Internet“ ist (Marx/Weidacher 2014: 65). Heyd (2016: 90) datiert den Beginn des Webs 2.0 auf das Jahr 2004, O'Reilly (2012: 32) etwas früher auf 2001. Der Begriff Web 2.0 beschreibt ,a notion of Internet usership that is more active, participatory, and collaborative.“ (Heyd 2016: 90) Heute ist die überwiegende Mehrheit aller Internetprodukte Teil des Webs 2.0. Die von Heyd als partizipativ und kollaborativ benannte Ausrichtung des Webs 2.0 rührt daher, dass Produkte des Webs 2.0 sich stärker auf die kollektive Intelligenz der Medienbenutzer ausrichten. Hier- 
bei sind die Benutzer nicht mehr wie im Web 1.0 passive Konsumenten, sondern es wird ihnen eine größere Mitwirkung eingeräumt (cf. O’Reilly 2012: 51; Marx/Weidacher 2014: 69f.).

Internet Memes sind demnach ein typisches Phänomen des Webs 2.0: Sie können einfach von verschiedenen Benutzerinnen erzeugt, individuell angepasst und geteilt werden. Ihre Entstehung und Verbreitung hängt von der für das Web 2.0 typischen kollektiven Intelligenz ab. Inwiefern auch Memes diese partizipative Eigenheit des Webs 2.0 vereinnahmen, beschreibt Milner (2016) wie folgt: „Internet Memes depend on collective creation, circulation, and transformation" (14). Milner greift mehrere Beispiele auf, in denen gezeigt wird inwiefern die Affordanzen ${ }^{3}$ verschiedener Plattformen des Webs 2.0 die Teilnahme und Verbesserung dieser Plattformen durch die Nutzer ermöglichen und ermutigen. Milner zeigt auf, wie in einem traditionelleren Medium entstandene Momente von den Usern der Plattformen des Webs 2.0 übernommen und neu kontextualisiert werden können, wodurch die traditionelleren Medien einen gewissen Kontrollverlust erfahren (cf. Milner 2016: 14f.). Er zeigt auf, dass die partizipative Eigenschaft des Webs 2.0 durchaus weitreichende Konsequenzen für die traditionellen Medien hat, was dazu führt, dass diese einen gewissen Machtverlust in Kauf nehmen müssen. Traditionelle Medien haben beispielsweise wenig Kontrolle darüber, ob und welche humoristischen Kommentare über ihre Inhalte in anderen Plattformen hergestellt werden.

So entsteht ein Phänomen, dessen Akteure als new audience (cf. Gillmor 2004), neues Publikum, bezeichnet werden können. Rosen beschreibt diese Akteure wie folgt:

The people formerly known as the audience are those who were on the receiving end of a media system that ran one way, in a broadcasting pattern with high entry fees and a few firms competing to speak very loudly while the rest of the population listened in isolation from one another and who today are not in a situation like that at all.

(Rosen 2012: 13)

Mit dem Aufkommen des Internets hat eine Auflösung dieses Machtmonopols der herkömmlichen Medien begonnen. Die frühere Zweiteilung zwischen Medienschaffenden und Medienkonsumierenden verschwimmt zunehmend. Blogs, Youtube-Kanäle und, im hier vorliegenden Fall, Kommentarfelder, ermöglichen es einer großen Menge an vormals medial passiven Zuschauern, sich selbst aktiv an Diskursen zu beteiligen, zu welchen ihnen vorher kein Zugriff gewährt war. Während gewisse Forscher das Risiko der Qualitätsminderung hervorheben (cf. Schlobinski 2005: 4), welches mit dieser neuen Form des Publikums einhergeht, so sehen andere es als eine Chance zum ,grassroots journalism“ (Gillmor 2004: Absatz 7), wo eine größere Anzahl an Menschen die Möglichkeit haben, an verschiedenen Diskursen teilzunehmen.

Gegen die Konzeptualisierung des neuen, aktiv mitdiskutierenden Publikums im Gegensatz zum früheren, passiven Publikum, ist einzuwenden, dass bereits vor dem Web 2.0 Medienkonsumenten gewisse Möglichkeiten hatten, als Produzenten aktiv zu werden. So existieren etwa in Zeitungen gedruckte Leserbriefe oder es können sich bei gewissen Radioprogrammen die Zuhörer per Telefon mitbeteiligen. Gleichzeitig ist das Web 2.0 nicht allen zugänglich:

\footnotetext{
${ }^{3}$ Tienken (2015: 36) beschreibt Affordanzen als „Nutzungsangebote der Umwelt (...) deren Bedeutungspotential allerdings von Nutzern auf verschiedene Weise erschlossen werden muss.“
} 
Zugriff zum Web 2.0 ist von Technologien abhängig, über welche immer noch nicht alle Menschen verfügen. Zensur erschwert zudem die Mitbeteiligung von Internetnutzerinnen in gewissen Ländern. Dennoch ist dieses neue Publikum in der hier vorgestellten Form dasjenige, welches die beiden Satireseiten mit ihren Memes ansprechen. Ein Publikum, das aktiv mitdiskutiert und nicht wie bei den äquivalenten Fernsehprogrammen von Heute Show und Extra 3 vor dem Bildschirm oder als Livepublikum passiv im Hintergrund verweilt.

\subsection{Nachrichtenparodie im Fernsehen und darüber hinaus}

Die Heute Show und Extra 3 können mit dem von Baym/Jones (2012) konzipierten Konzept news parody (im weiteren Text als Nachrichtenparodie übersetzt) beschrieben werden. Nachrichtenparodien dienen als ,the launching point for critique of the actual sources of popular information, an examination, in varying degrees of acuity, of news practices [...]" Baym/Jones 2012: 5). Zweck von Nachrichtenparodien ist einerseits, Kritik an der Textsorte „Nachrichten“ und deren Kommunikationsstil zu üben, andererseits aber auch eine Kritik am breiteren öffentlichen Diskurs zu äussern, welcher auch in herkömmlichen Nachrichten besprochen wird (ibd.). Extra 3 wie auch Heute Show entsprechen dieser Beschreibung: Sie sind ähnlich gestaltet wie eine Nachrichtensendung und befassen sich einmal die Woche im Rahmen einer halben Stunde mit den politischen Geschehnissen Deutschlands und der ganzen Welt (cf. Kleinen-von Königslöw/Kehl 2013). Sie stellen einen ,hybrid blend of news, political conversation, and comedy“ (ibd.: 6) dar, welche das momentane Weltgeschehen satirisch kommentiert. Die Begriffe „Nachrichtenparodie“ und „Satire“ schließen sich gegenseitig nicht aus. Vielmehr bezeichnen Baym/Jones (2012: 4) Nachrichtenparodie als einen Sammelbegriff für variierende Sendungen, welche allesamt Humor benutzen, um das Tagesgeschehen in Politik und der Gesellschaft satirisch zu kommentieren und kritisieren. Im Allgemeinen sind Nachrichtenparodien, ebenso wie die traditionellen Nachrichten, eng an das Medium Fernsehen gebunden. Mittlerweile haben aber viele Nachrichtenparodien auch eine Onlinepräsenz auf sozialen Netzwerkplattformen wie Facebook und Twitter aufgebaut, wo neben Zitaten und Ausschnitten aus den Fernsehsendungen auch Inhalte publiziert werden, welche in dieser Form nicht in der Fernsehsendung existierten.

Durch diese Auslagerung der Nachrichtenparodie in ein neues Medium wird der Nährboden gesetzt für potentielle Hybridisierungen. Hybridisierungen lassen sich in ,,praktisch allen Bereichen massenmedialer Kommunikation nachweisen“ (Hauser/Luginbühl 2015: 7) und beschreiben im linguistischen Kontext den Prozess, in welchem aus bereits existierenden Texten oder Textsorten Merkmale übernommen und neu zusammengesetzt werden. Durch diese Mischung unterschiedlicher Textsorten kommt es zu einer „Kombination und Verflechtung unterschiedlicher und teilweise widersprüchlicher Codes“, was in einer „Herausbildung neuer ,emergenter" kultureller Texte“ (ibd.: 8) resultiert. Bakhtin beschreibt die Hybridisierung als „mixture of two social languages, an encounter, within the arena of an utterance“ (Bakhtin 1981: 358). Es vermischen sich also im Hybridisierungsprozess zwei Sprachen oder Sprechweisen (cf. Tienken 2015: 38). Im hier untersuchten Zusammenhang scheint eine Entstehung von Hybridisierung möglich und sogar wahrscheinlich, da hier zwei verschiedene Medien (Fernsehen und Internet) miteinander kollidieren und sich somit neue Texte bilden könnten, welche als Hybridisierungen kategorisiert werden können. 


\section{3 Überlegungen zu Diskurs und Macht}

Die medialen Aushandlungen zum Thema G-20-Gipfel können als Diskurs konzeptualisiert werden Diskurse betrachte ich in Anlehnung an Foucault als ,,auf ein Thema bezogene Textoder Aussagenverbünde [...], eine Menge von Aussagen, die einem gemeinsamen Formationssystem angehören, das nach bestimmten Regeln funktioniert.“ (Dreesen/Kumiega/Spieß 2012: 76) Es ist ein grundlegendes Merkmal eines Diskurses, dass durch ihn etwas Neues produziert wird, etwa Texte oder Effekte. Der Diskurs ist aber keine ,über allem Gesellschaftlichen schwebende Struktur, kein System, das es unabhängig von den Menschen gäbe. Im Gegenteil: Diskurse sind Resultat menschlicher Tätigkeit, gleichsam Resultante des gesamtgesellschaftlichen Tuns der Subjekte [...]“ (Jäger 2009: 78).

Eng mit dem Foucault'schen Diskursbegriff verbunden ist auch das Konzept der Macht. Mit Macht ist nach Foucaults Konzeptualisierung keine restriktive Macht gemeint (cf. Dreesen/Kumiega/Spieß 2012: 9) sondern vielmehr „ein Beziehungsgefüge, welches eine Gesellschaft durchzieht“" (Spitzmüller/Warnke 2011: 74). Metaphorisch beschreibt Foucault Macht als ein Netz, welches ,den ganzen sozialen Körper überzieht und nicht so sehr als negative Instanz" (Foucault 1978: 35). Macht wird durch den Diskurs produziert, wodurch der Diskurs selbst die Macht ist, welche man zu bemächtigen sucht. In diesem Zusammenhang sind Medien und Medialität wichtige Werkzeuge, sie wirken als ein ,produktives Bedingungsgefüge“ (Dreesen/Kumiega/Spieß 2012: 10). Auch der Diskurs über den G-20 Gipfel wurde zu grossen Teilen durch verschiedene Medien als Wirklichkeit konstruiert und die Machtverhältnisse in und um das Ereignis wurden auf diesem Wege produziert.

Der G-20 Gipfel sowie die Demonstrationen im Vorfeld und Nachhinein können als Teile eines diskursiven Ereignisses verstanden werden. Ein diskursives Ereignis bezeichnet ein Ereignis, welches Anstoss zu einem Diskurs gibt. Diskursive Ereignisse unterscheiden sich von sogenannt realen Ereignissen dadurch, dass sie erst durch den Diskurs über das Ereignis zu existieren beginnen (cf. Jäger 2009: 132). Das Ereignis G-20 Gipfel 2017 kann also insofern als ein diskursives Ereignis eingeordnet werden, als dass es sich bei ihm um menschliche Aktivitäten handelt, welche dann einen Diskurs auslösen, der in verschiedenen Medien geführt wird.

Auch lange Zeit nach dem Gipfel lassen sich immer noch Spuren des G-20-Diskurses finden. So wird etwa im folgenden Twitter-Post noch im Juni 2018 der G-20-Gipfel als Anknüpfungspunkt für Kritik an rechtsextremistischen Ausschreitungen genutzt, indem ironisierend die vieldiskutierten „Flaschenwürfe bei G20“ als belangvoller beschrieben wird als eine Feindesliste von Rechtsextremen. 


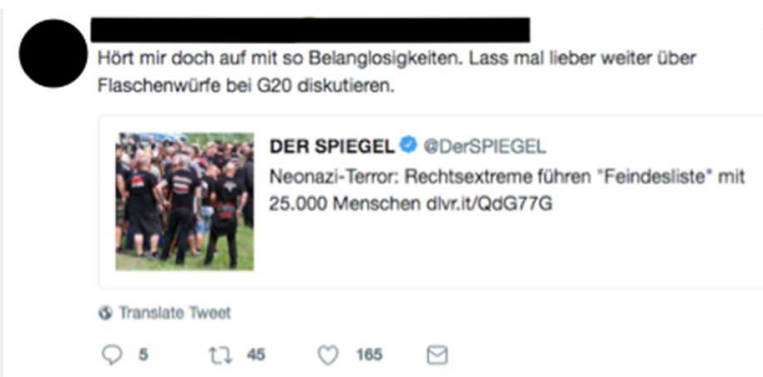

Abbildung 4: Beispiel Diskurs G-20 Gipfel, geteilt auf Twitter 30.6.2018

Das diskursive Ereignis G-20 Gipfel umfasst demnach nicht nur einen einzigen einheitlichen Diskurs, sondern besteht aus verschiedenen Diskurssträngen, sogenannten „Abfolgen von Diskursfragmenten mit gleicher Thematik“ (Jäger 2009: 117). Zu einem dieser Diskursstränge gehört auch der satirische Diskurs über den G-20 Gipfel und, als Subkategorie dieses Diskursstrangs, die Memes der beiden großen Satiresendungen Deutschlands. Die Memes sind Manifestationen des diskursiven Ereignisses, welche somit dazu beitragen, dass der G-20 Gipfel als diskursives Ereignis überhaupt erst existiert.

In dieser Studie wird Diskurstheorie auch als Methode der Korpusgenerierung benutzt. Der G-20 Gipfel 2017 ist ein diskursiv klar begrenztes Ereignis, zu welchem eine übersichtliche Anzahl Memes existiert, welche thematisch miteinander verbunden sind. Diese werden als eine bestimmte Manifestation des satirischen Diskursstrangs zum G-20 Gipfel betrachtet. Der untersuchte Diskursstrang ist jedoch eng mit dem übergreifenden Diskurs verbunden. Die Memes meines Korpus und deren Kommentare entstehen nicht in einem Vakuum und sind deutlich mit mehreren Diskursen verbunden. Deshalb ist es von Wichtigkeit, den diskursiven Zusammenhang der Memes (also den G-20 Gipfel und die Berichterstattung zu ihm) miteinzubeziehen.

\section{Satire und Satireverhandlungen in Memes und Kommentaren}

\subsection{Satire und Memes}

Nach Brummacks Konzeptualisierung von Satire als ästhetisch sozialisierte Aggression können die untersuchten Memes als satirisch interpretiert werden. Sie beinhalten allesamt eine in einem sozialen Rahmen (also in den untersuchten sozialen Netzwerkplattformen) geäusserte Aggression, welche sich durch eine demonstrierbare Ästhetik auszeichnet. Die in den Memes vorkommenden „Opfer“ dieser satirischen Aggression (cf. Brummack 1971: 282) sind die Organisatoren des Gipfels (cf. Abbildung 8), die gewalttätigen Demonstranten (cf. Abbildung 25), die am Gipfel teilnehmenden Machthaber (cf. Abbildung 11), Polizei und Justiz (cf. Abbildung 27), sowie der Diskurs über den Gipfel (cf. Abbildung 10). In untersuchten Kontext erscheint Aggression als mehr oder weniger implizite spotthafte Kritik. Hier kommt auch die soziale Ebene des Satirebegriffs zum Zug: Alle erfassten Memes vermitteln in ihrem Inhalt eine Art konstant gehaltenes Wertesystem, welches dem Publikum vermittelt werden soll. Dieses Wertesystem bleibt für beide Sendungen gleich. Gleichsam der ursprünglichen Nachrichtenparodie beziehen sich die Inhalte der Memes auf gesellschaftliche Themen, im hier vorliegenden Fall zum G-20 Gipfel. Es werden die gleichen Themen aufgegriffen, welche auch den nicht-satirischen Diskurs zum G-20 Gipfel prägen. 
Im Gegensatz zu anderen satirischen Texten kombinieren die Memes in der untersuchten Datensammlung Bild und Schrifttext zu einem satirischen Kommentar zum G-20 Gipfel. Ein Beispiel für ein typisches Meme dieser Datensammlung ist folgendes:

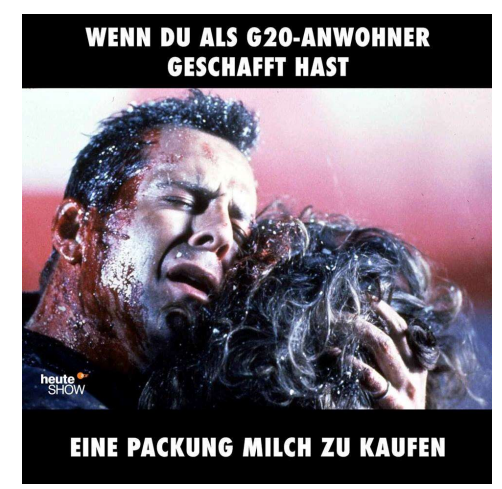

Abbildung 5: G20-Anwohner-Meme

Allein gehalten wären sowohl Text wie auch Bild (welches einen Ausschnitt aus dem Actionfilm Die Hard abbildet) nicht humoristisch. In ihrer Kombination erwirkt allerdings die überzogene Dramatik einen humoristischen Effekt: Der Einkauf im Supermarkt wird wegen der Unruhen zum Spiessrutenlauf oder zur Heldentat, ähnlich der Die Hard-Filme, uminterpretiert. Hier zeigt sich die Wichtigkeit der Kombination von Bild und Text für das Verständnis des satirischen Inhalts und den humoristischen Ausdruck.

\subsection{Memes und ihre Formate}

Formal greifen die gesammelten Memes auf verschiedene Formen zurück, in denen sich Memes manifestieren können. Einige Memes können vom Format her als Image Macro eingeordnet werden (für Erklärung siehe 1.1). Bei anderen in der Datensammlung vorkommenden Memes handelt es sich um sogenannte thematische Bilder, wo der Bildteil des Memes „direkt mit der im Internet-Meme angesprochenen Thematik verbunden [ist]“ (Weidacher 2019: 174). Dazu zählen also Memes, welche Bildmaterial nutzen, das mit dem G-20 Gipfel oder dessen Akteuren in direktem Zusammenhang steht. Daneben gibt es in meiner Sammlung noch ein Meme, welches keinen Text, sondern nur Bildmaterial verwendet und zwei, in denen das Bildmaterial minimal gehalten ist (cf. Abbildung 6).

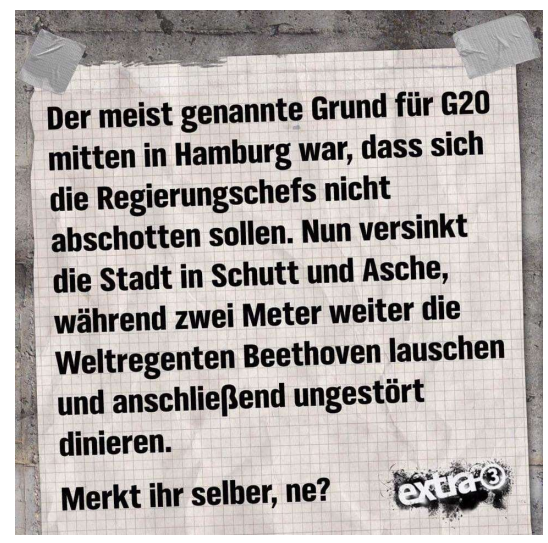

Abbildung 6: „Merkt ihr selber“"-Meme

Diese zwei Memes weichen am stärksten vom klassischen Image Macro-Format ab und es kann diskutiert werden, ob es sich bei ihnen überhaupt noch um Memes handelt. Sie werden 
allerdings im gleichen Zusammenhang geteilt wie auch die anderen Memes und erfahren die gleiche Verbreitung durch Likes, Kommentare und Shares. Obwohl der Bild-Teil eher im Hintergrund steht, so ist dieses Meme doch immer noch ein multimodaler Text. Dies zeigt sich dadurch, dass der Text in Abbildung 6 als ein an eine Wand geklebtes Plakat dargestellt wird, dem bildlichen Hintergrund also doch eine gewisse Miteinwirkung eingeräumt werden sollte. Es handelt sich nicht bloß um einen Text auf weissem Hintergrund, sondern es gibt gewisse visuelle Elemente, die ebenfalls zur Bedeutung beitragen. Im zweiten, praktisch bildfreien Meme, spielen die nonverbalen Elemente eine noch grössere Rolle zum Verständnis des Memes:

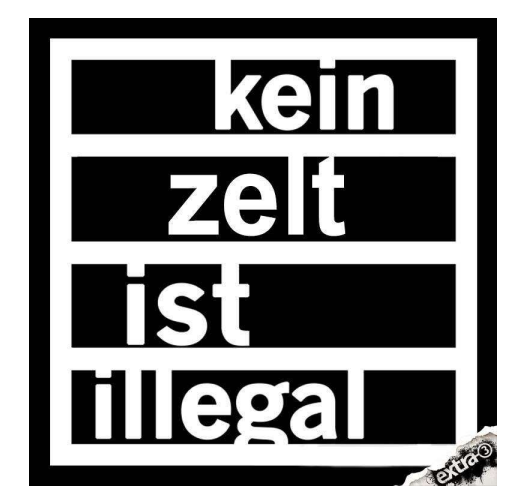

Abbildung 7: „Kein Zelt ist illegal“-Meme

Die Schriftart, Farben sowie das Format verweisen intertextuell auf den im Jahr 1988 geprägten „Kein Mensch ist illegal“-Slogan und dessen typographische Ausgestaltung (cf. Wikipedia s. v. kein mensch ist illegal). Es ist also nicht nur die Schrift, sondern es sind durchaus auch die bildlichen Elemente, welche die satirische Kritik des Memes offenbaren.

Die Memes, welche von der Gestaltung her nicht genau in das schematische Image-MacroFormat passen, könnten als Hybridisierung zwischen den Textsorten Satiresendung und klassischem Image Macro kategorisiert werden. So wird das klassische Image Macro-Format zugunsten der satirischen Botschaft der Satiresendung angepasst, wie es etwa in Abbildung 8 beobachtet werden kann. Satirische Memes als Hybridisierungen mit unterschiedlich starkem Hybridisierungsgrad zu kategorisieren, erklärt gewisse Differenzen zwischen den Memes in meiner Datensammlung und den klassischen Image Macros. Gleichzeitig erfordert diese Erkenntnis weiterführende Forschung zur genaueren Einstufung der Memes als Hybridisierung. Es müsste weiter abgeklärt werden, welche zwei Sprachen oder Sprechweisen sich in den untersuchten Memes vermischen und inwiefern diese als Sprache oder Sprechweise kategorisiert werden können. Weitere Forschung müsste genauer überprüfen, inwiefern es sich bei Satiresendungen und Memes um Sprachen/Sprechweisen handelt und inwiefern durch eine Kombination dieser zwei eine Hybridisierung zustande kommt.

\subsection{Ironie in der Ausgestaltung von satirischen Memes}

Ironie ist ein in meiner Datensammlung vielfach angewandtes Stilmittel. So ist bei vielen der Aussagen in den Memes zu vermuten, dass diese nicht ernst gemeint sind. ${ }^{4}$ Ebenfalls setzen

\footnotetext{
${ }^{4}$ Eine intuitive Interpretation der Memes als „,nicht ernst gemeint“ ist in diesem Zusammenhang unvermeidbar, da sich die Frage nach der Seriosität empirisch nicht nachweisen lässt.
} 
sie vom Leser ein spezifisches Vorwissen voraus, sowohl was das Geschehen des G-20 Gipfels angeht, wie auch im Bezug auf die in den Memes vorhandenen Bewertungen. Es wird vorausgesetzt, dass man der medialen Berichterstattung zum G-20 Gipfel und auch Vorkenntnisse zu lokalen Gegebenheiten Hamburgs hat (wie etwa der Hamburger Hafengeburtstag in Abbildung 8) Ironie manifestiert sich beispielsweise in den Memes dadurch, dass der SchriftText in klarem Kontrast zum Bild-Text steht.

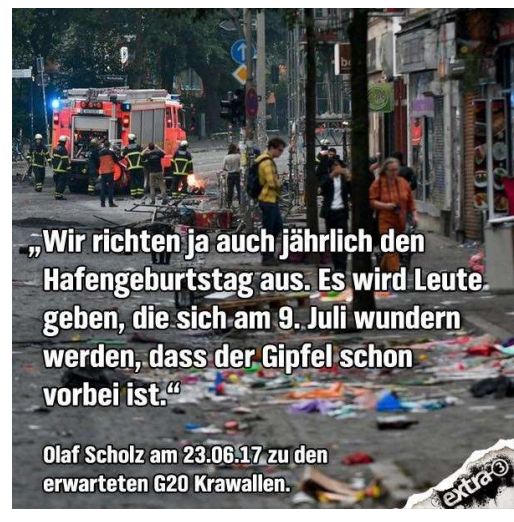

Abbildung 8: Zitat Scholz-Meme

Dieses Meme weicht in seiner Form deutlich vom herkömmlichen Image-Macro-Format ab, indem der Text in der Mitte platziert ist und die übliche Punchline sich nicht im Text befindet. Diese wird aber ersichtlich, wenn der Schrifttext mit dem den Hintergrund bildenden Bild in Kontext gesetzt wird. Die zitierte Aussage des damaligen Hamburger Bürgermeisters Olaf Scholz, welche den damals noch bevorstehenden G-20 Gipfel mit dem alljährlichen Volksfest des Hafengeburtstags gleichsetzt, steht in starkem Kontrast zum Bild, welches die tatsächliche Zerstörung in der Stadt zeigt. Dadurch wird die Aussage Scholz' ironisiert. Mehrere Memes manifestieren Ironie auf diese Weise. In anderen Memes stehen zwar Text und Bild nicht in direktem Kontrast zueinander, tragen aber gemeinsam zur Implikatur bei, dass der Text ironische Aspekte beinhaltet, wie etwa in folgendem Beispiel, wo wiederum ein Zitat von Olaf Scholz zur Ironisierung beiträgt.

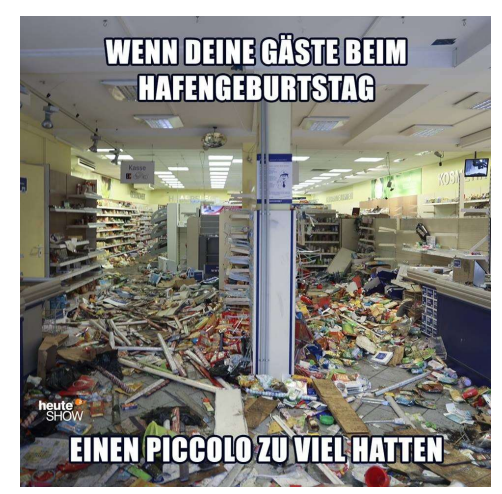

Abbildung 9: „Gäste beim Hafengeburtstag“-Meme

Verwendet wird hier ein Foto von einem verwüsteten Ladengeschäft, welches die Zerstörung nach dem G-20 Gipfel repräsentieren soll. Der Schrifttext bezieht sich ebenfalls auf Olaf Scholz' berühmt gewordene Aussage zum Hamburger Hafengeburtstag. Mit dieser Aussage begründete der damalige Hamburger Bürgermeister seine Zuversicht, dass der G-20 Gipfel in Hamburg problemlos verlaufen werde. Somit wird im Meme Scholz' Aussage ins Absurde 
übertragen, indem ironisierend die Zerstörung durch Demonstranten mit einer feuchtfröhlichen Feier am Hafengeburtstag gleichgesetzt wird. Das Bildelement steht in diesem Fall nicht in Kontrast zum Text, trägt jedoch dazu bei, dass die Originalaussage Olaf Scholz' ins Absurde übertragen wird. Die Bildelemente können also entscheidend zum Ironie-Verständnis beitragen, oder, wie im untenstehenden Beispiel, bloss illustrierend mitwirken.

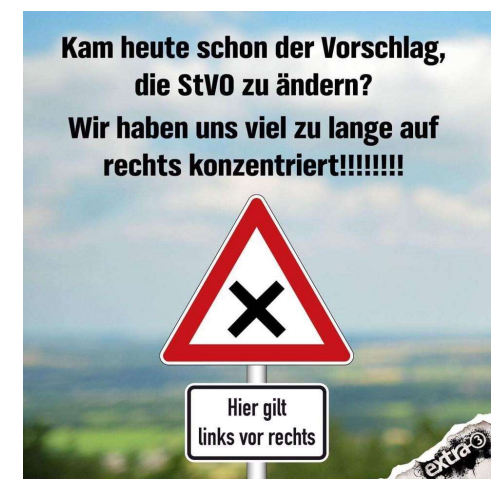

Abbildung 10: Links vor Rechts-Meme. Satirischer Kommentar zum Diskurs über Links- vs. Rechtsextremismus nach dem G-20 Gipfel

Die angewandte Ironie setzt von ihren Lesern vielfach ein Vorwissen über die Einstellungen der Produzentinnen der Memes voraus. In folgendem Beispiel muss der Leserin etwa klar sein, dass die Produzenten der Memes Merkel und Trump keineswegs für vertrauenswürdige Experten halten. Diese Lesart wird den Betrachtern der Memes dadurch nahegelegt, dass Merkel und Trump auf dem Bild ein Gesicht mit unvorteilhaftem Mienenspiel zeigen, was sie bildlich keineswegs als Experten markiert.

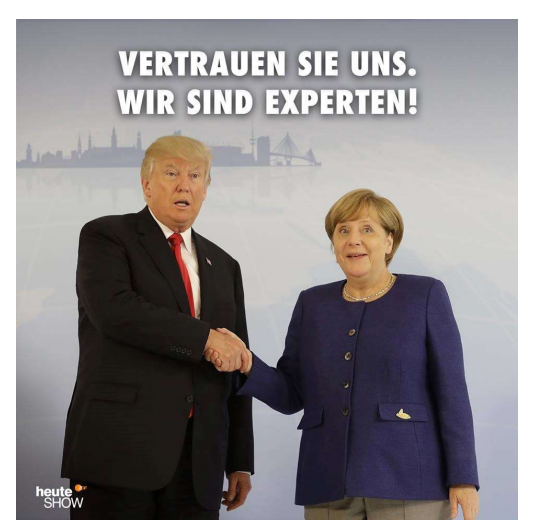

Abbildung 11: „Wir sind Experten"-Meme

Ironie kann in der überwiegenden Mehrheit der Memes dieser Studie gefunden werden. Sie ist das rhetorische Werkzeug, welches die Produzenten der Memes für ihre satirische Kritik an verschiedenen Akteuren unter dem G-20 Gipfel nutzen. Die Ironie manifestiert sich in den gesammelten Memes jeweils unterschiedlich, sie ist jedoch immer das Hauptwerkzeug, um an den verschiedenen Akteuren während des G-20 Gipfels satirische Kritik zu üben.

\subsection{Internet Memes als Manifestation der Nachrichtenparodie im Web 2.0}

Die im Web 2.0 gegebenen partizipativen Aspekte ermöglichen, dass das Satireformat der Nachrichtenparodie neue Formen annehmen kann und sich Phänomene entwickeln können, welche zuvor in diesem Ausmass nicht existierten. Das neue Format der Nachrichtenparodie 
kann wie folgt konzeptualisiert werden: Das Meme stellt die satirische Vorführung dar, welche im Medium Fernsehen die Performance der Moderatoren ist. Die Kommentarsektion kann mit dem in der Fernsehsendung anwesenden Publikum parallelisiert werden. Hier zeigt sich, wie Internet Memes eine Manifestation der Nachrichtenparodie im Web 2.0 darstellen können: Sie sind ein Werkzeug für die Produzenten der Fernsehsendungen, um auch im Web 2.0 relevant zu sein. Zwar bringen die Affordanzen des Webs 2.0 einige Herausforderungen mit sich (siehe 4.5). Dennoch bringt dieses Medium den Satireproduzenten erhebliche Vorteile: Sie können sich auf diese Weise am Diskurs zum G-20 Gipfel beteiligen, obwohl beide Sendungen offiziell Sommerpause haben. Ausserdem bietet das Web 2.0 die Möglichkeit, ein neues, jüngeres Publikum anzusprechen, welches seine Unterhaltung nicht aus dem Fernsehen, sondern aus dem Internet bezieht. Memes sind also eine Manifestation des Phänomens, dass Nachrichtenparodien ihre Satire immer mehr aufs Internet ausweiten.

\subsection{Die Affordanz Kommentieren und dessen Effekte}

Das Medium Internet bietet für die hier vorliegende satirische Nachrichtenparodie eine Vielzahl an Affordanzen, welche im Medium Fernsehen nicht gegeben waren: So etwa die Möglichkeit, rund um die Uhr Beiträge zu publizieren oder neben Filmabschnitten auch Bild- oder Textbeiträge veröffentlichen zu können und nicht zuletzt auch die geringeren Kosten einer Onlinepräsenz im Gegensatz zu einer Fernsehproduktion. Des Weiteren bietet das Internet, insbesondere auf den sozialen Netzwerken wie Facebook, eine größere Möglichkeit zur Partizipation des Publikums. In den Kommentarspalten können sich die Leser wertend zu den vorhandenen Memes äußern oder selbst satirische Texte verfassen. Dies birgt jedoch auch eine Tücke: Aufrufe zu oder Androhungen von Gewalt sind auf beiden Plattformen vorhanden. Obwohl beide Seiten in ihren Netiquette-Beschreibungen zum rücksichtsvollen Umgang mit den anderen Nutzern aufrufen (cf. ZDF Heute Show auf Facebook: Netiquette und NDR: Kommentarrichtlinien) wird diesem Gebot oft nicht Folge geleistet. Mehrheitlich werden diese Regelbrüche von den Betreiberinnen nicht gelöscht. Ein Beispiel hierfür ist folgender Topkommentar, welcher unter dem Anwohner-Hamburg-Meme (Abbildung 5) gefunden wurde:

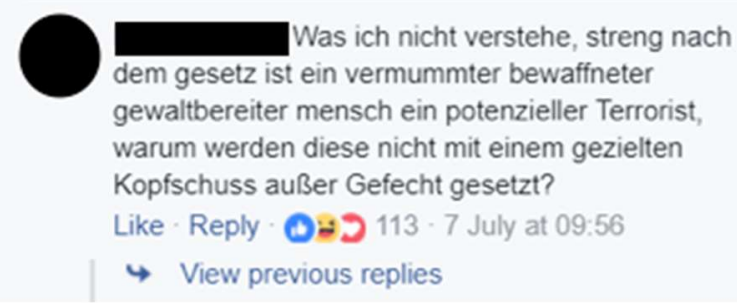

Abbildung 12: Kommentar unter Meme 7, „Anwohner Hamburg“-Meme

Insbesondere, wenn sich die Diskussion um die Randalierer in Hamburg dreht, sind Aufforderungen und Wünsche nach gewalttätigerem Umgang mit diesen zu verzeichnen. Neben tatsächlichen Gewaltaufrufen, lässt sich in den Kommentaren auch regelmässig verbale Gewalt finden. Schwarz-Friesel und Reinharz beschreiben verbale Gewalt als ,eine destruktive Form der Machtausübung“, welche das Ziel hat, „den/die Anderen zu kränken, zu beleidigen, auszugrenzen [...]“ (Schwarz-Friesel/Reinharz 2013: 40). Die Konzeptualisierung von verbaler Gewalt fusst auf dem Grundsatz, dass Sprache ein Handlungsinstrument ist, welches „wie eine Waffe benutzt werden [kann], um Menschen Schaden zuzufügen, [...]“ (ibd.: 31) und 
dass Sprache daher als Handlungsmaterial das Potential hat, „Menschen kognitiv wie emotional zu verletzen“ (ibd.: 41). Der in Abbildung 24 enthaltene Kommentar ,was will man von einem ,Abdul' auch anderes erwarten“ ist ein Beispiel für die in den gesammelten Kommentaren vorkommende verbale Gewalt. Die Aussage, dass von einem anderen Kommentator allein aufgrund seines Namens nichts zu erwarten sei, ist nicht nur eine beleidigende Äusserung, sondern erhält durch den arabischen Ursprung des Namens $A b d u l$ auch eine rassistische Konnotation und kann daher klar als verbale Gewalt klassifiziert werden. Unklar ist die Signifikanz der Kothaufen-Emojis, welche den Kommentar begleiten und möglicherweise die Botschaft abschwächen oder eine gewisse Selbstironie erkennen lassen sollen. Da deren Bedeutung jedoch nicht explizit erkennbar ist, kann nicht davon ausgegangen werden, dass der Kommentar durch sie eine andere Bedeutung erhält.

Grundsätzlich ist verbale Gewalt, wie sie in der Datensammlung existiert, ein häufiges Vorkommnis auf Facebook, wofür die Plattform auch ständig viel Kritik erhält (cf. z. B. The Verge zu Facebook-Posts bezüglich Sri Lanka und Myanmar). Betreiber von Facebookseiten haben zwar die Möglichkeit, Kommentare zu löschen, aber angesichts der grossen Anzahl Kommentare auf den beiden hier untersuchten Facebook-Seiten ist ein ständiges Moderieren der Kommentarsektion kaum praktikabel und möglicherweise für die Betreiber auch nicht erstrebenswert. Denn grundsätzlich ist erhöhte Partizipation für Seiten auf sozialen Netzwerken immer gewinnbringend, da durch sie der Traffic auf der Seite zunimmt. Dies kann ebenfalls eine Erklärung sein, weshalb die Kommentare auf den auf den Seiten der Heute Show und Extra 3 kaum moderiert zu sein scheinen.

Diese wiederkehrende verbale Gewalt verweist auf eine andere, negative Seite des neuen Publikums. Die Möglichkeit des neuen Publikums, Medien nicht nur zu konsumieren, sondern auch zu produzieren ermöglichen eben auch, dass es zu Beleidigungen, Ausgrenzungen und Diskriminierungen kommt, welche als verbale/digitale Gewalt oder als Cybermobbing (cf. Marx 2017) kategorisiert werden kann. Dies bietet Gelegenheit für „eine Form psychischer Gewalt, die von Initiatoren vornehmlich verbal realisiert und über technologische Applikationen einem in der Größe variierenden Kreis von Zeug_inn/en zugänglich gemacht wird“" (ibd.: 12). Während in anderen Medien verbale Gewalt entweder stärker unterbunden wird oder aber von einem kleineren Publikum rezipiert wird, bietet das Internet eine große und weitgehend unzensierte Plattform für dieses Phänomen. Das Erscheinen dieses Phänomens auch in der hier vorliegenden Datensammlung zeigt auf, dass das Phänomen des neuen Publikums neben positiven Konsequenzen wie der Demokratisierung der Medienproduktion auch problematische Aspekte aufweist.

Gleichzeitig drücken die Rückantworten zu diesen Kommentaren grösstenteils Entrüstung und Entsetzen über die Gewaltaufrufe aus. Dies zeigt sich auch in den Rückantworten zur in der obigen Abbildung gestellten rhetorischen Frage, weshalb vermummte Randalierer nicht erschossen würden. 


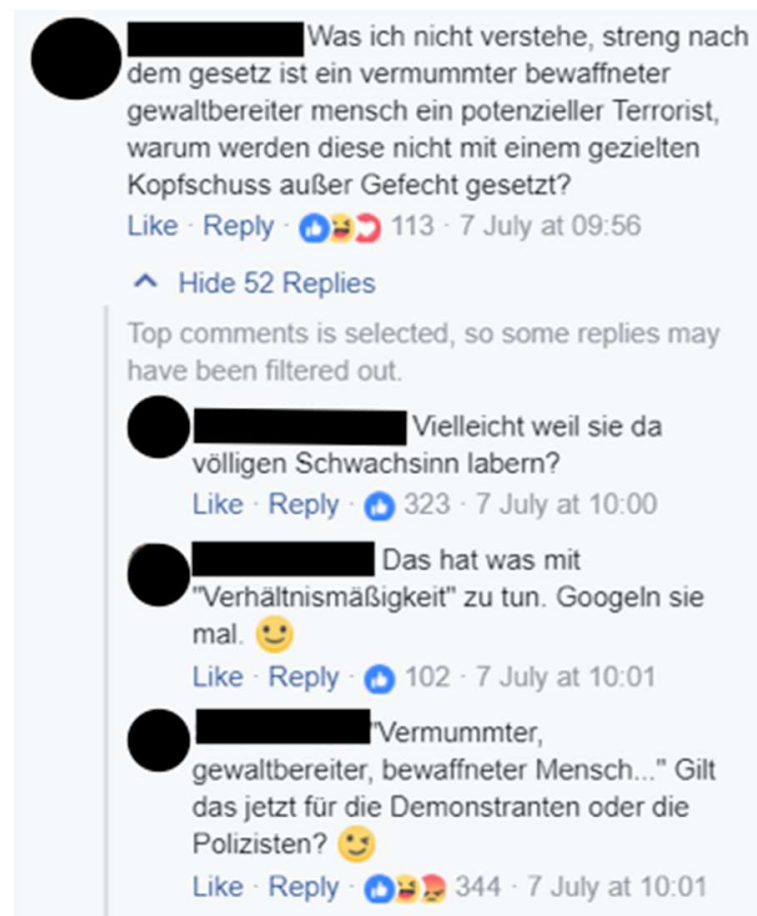

Abbildung 13: Kommunikativer Kontext zu Kommentar in Abbildung 12

Die Aussage des Topkommentars wird als „völliger Schwachsinn“ und „unverhältnismässig“ bezeichnet. Des Weiteren erhält die Rückantwort (Abbildung 13 zuunterst), welche ironisch fragt, ob mit den im Ursprungskommentar erwähnten Terroristen die Polizei oder die Demonstranten gemeint seien, mehr Likes als der eigentliche Topkommentar. Von den 52 Rückantworten, welche der Kommentar erhält, ist die überwiegende Mehrheit ablehnend zur Ansicht im Topkommentar eingestellt. Aufforderungen zur Gewalt erhalten also von anderen Kommentierenden starke Kritik.

\subsection{Die Kommentierenden als „neues Publikum“}

Eine Analyse der Top Kommentare (siehe Kapitel 2) auf der Wortebene zeigt, dass sich in folgende Gruppen einteilen lässt:

1. Zustimmende Kommentare

2. Ablehnende Kommentare

3. Weder zustimmende noch ablehnende Äusserungen zum Meme

Durch affirmativen oder kritischen Sprachgebrauch werden Kommentare für Gruppe 1, respektive 2, identifiziert. In die dritte Gruppe gehören Äusserungen, welche sich weder zustimmend oder ablehnend zum thematisierten Meme oder der Sendung äussern. Folgendes Diagramm zeigt die Menge der Kommentare in den drei Kategorien an: 


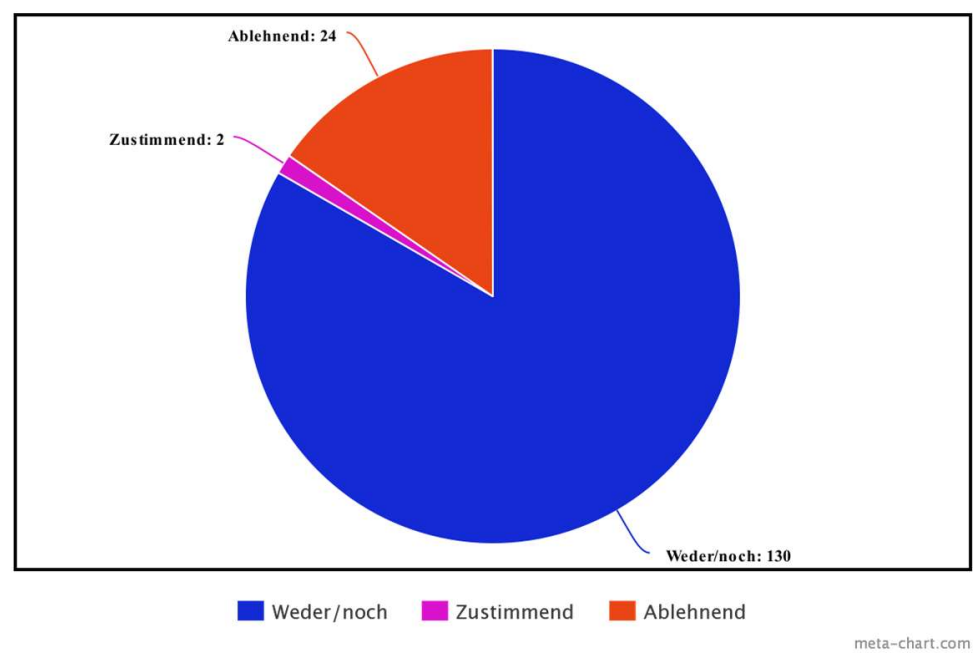

Abbildung 14: Kuchendiagramm Top Kommentare

Die zustimmenden Kommentare sind deutlich in der Unterzahl, nur zwei der im Datenset gefundenen Topkommentare lassen sich als solche kategorisieren. Dies kann damit zusammenhängen, dass Zustimmung zum Inhalt meist in Form von Likes oder Emojis, also Herzchen oder Lach-Reaktionen, ausfällt. Zustimmende Kommentare zeigen Sympathie für die Sendungen, etwa dadurch, dass nach dem nächsten Sendetermin gefragt wird, wie etwa in diesem Beispiel aus Meme Nr. 1, worauf die Betreiber der Facebookseite auch tatsächlich darauf reagieren:

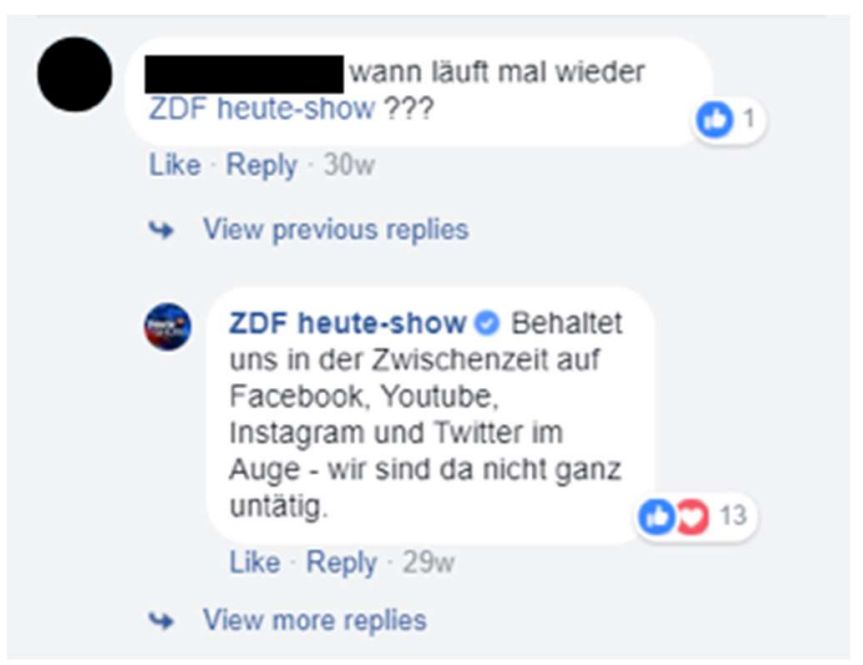

Abbildung 15: Beispiel zustimmender Topkommentar aus Kommentarsektion

Die ablehnenden Kommentare sind eine bedeutend grössere und eher heterogene Gruppe. So wird teilweise die politische Ausrichtung der Sendung oder des Memes kritisiert oder es wird der Sendung schlechter Geschmack vorgeworfen. Eine weitere, oft geäusserte Kritik ist der Vorwurf einer Verschwendung von Steuergeldern. Beide Sendungen werden auf öffentlichrechtlichen Fernsehsendern gezeigt, was die Basis dieses Arguments bildet. Die Kommentierenden verbinden also die Publikation der Memes direkt mit den von ihnen bezahlten Fernsehgebühren. 


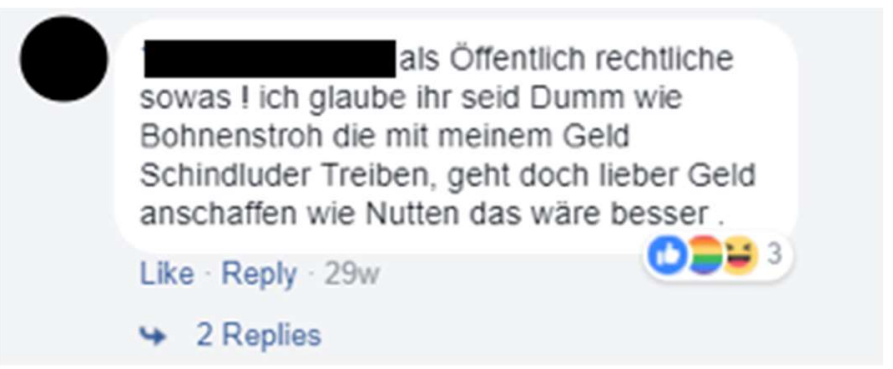

Abbildung 16: Unter ,Ehe für alle“-Meme

Die Kommentare, welche die Verbindung zum gebührenfinanzierten Fernsehen herstellen, zeigen einen relevanten Bezug zum Konzept des neuen Publikums auf. Die Kommentierenden sehen die Memes als eine Verschwendung von Steuergeldern und tun dies auch dementsprechend kund. Die Kommentierenden sehen sich also zu einem gewissen Grad als Finanzierungsbeteiligte und deshalb berechtigt, gewisse Erwartungen an das Produkt zu stellen.

Während die ablehnenden Kommentare zahlreicher sind als die zustimmenden, so wird doch in den Rückantworten zum Originalkommentar gekontert. Bei Vorwürfen zu schlechtem Geschmack kommt etwa häufig die Entgegnung, dass es sich bei dem Inhalt um Satire handelt, was als Rechtfertigung für einen provokanteren Umgangston angesehen wird. Ein Beispiel hierfür ist das titelgebende Zitat „Satire Junge SATIREEEE“, welches eine Rückantwort auf einen kritischen Kommentar darstellt.

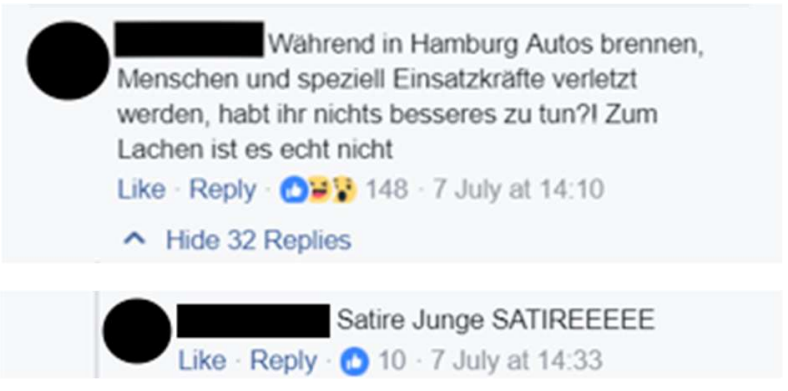

Abbildungen 17 und 18: Kritischer Topkommentar im „Drei Plagezeichen“-Meme und dessen titelgebende Rückantwort

Ein anderes Beispiel für das Aushandeln von Satire ist folgendes:

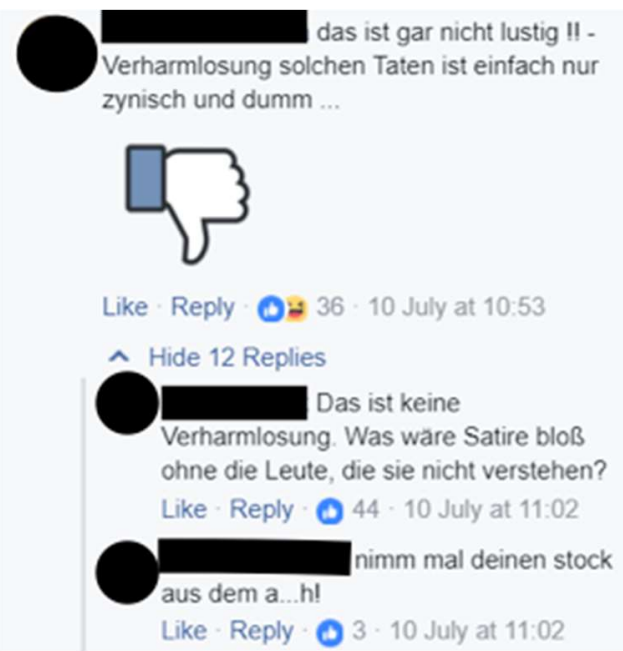

Abbildung 19: Unter „Gäste Hafengeburtstag“-Meme (Abbildung 9) 
Ausgangspunkt für solche Hinweise auf die satirische Gesinnung der Memes sind Kommentare, welche den Inhalt als nicht lustig oder geschmacklos bezeichnen. Dem Verfasser des ablehnenden Kommentars wird daraufhin Verklemmtheit (er solle seinen „stock aus dem a...h“ nehmen) oder Ignoranz vorgeworfen. Teilweise findet dann über mehrere Rückantworten hinweg eine Verhandlung darüber statt, was als Satire kategorisiert werden darf und welche Eigenschaften satirische Texte haben dürfen. Wie im obenstehenden Beispiel kommt es dabei zu Aussagen, die Kritiker hätten die Satire schlichtweg nicht verstanden. Kern dieser Aussagen ist oftmals, dass für Satire nicht dieselben Regeln gelten wie für andere Genres. So darf Satire provokante Aussagen machen, ohne dass diese als beleidigend oder unmoralisch kritisiert werden dürfen. Deshalb ist der Hinweis auf das Genre ein oft gebrauchtes Argument, um allfälliger Kritik an schlechtem Geschmack zu begegnen.

Diese Auseinandersetzungen mit dem Genre Satire offenbaren aus theoretischer Sicht zwei interessante Aspekte: Erstens lässt sich das Aushandeln eine Ethno-Kategorisierung quasi online beobachten. So haben viele Kommentierende unterschiedliche Ansichten darüber, welche Eigenschaften Satire ausmacht und verhandeln diese untereinander.

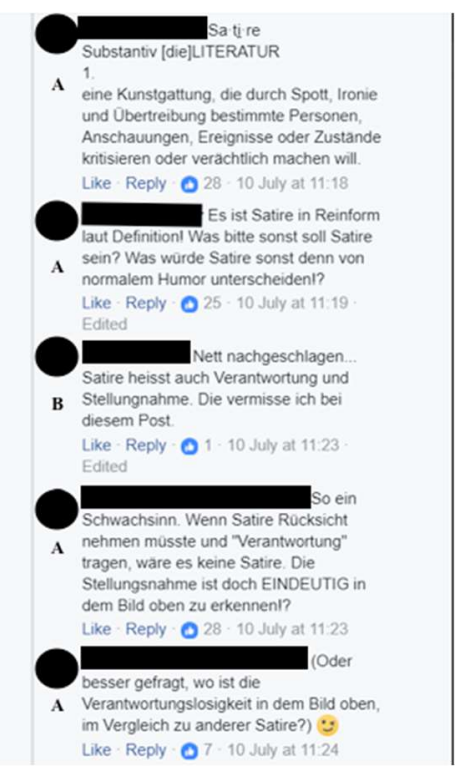

Abbildung 20: Unter „Gäste Hafengeburtstag“-Meme, A und B weisen die Gesprächsteilnehmer aus

Ethno-Kategorisierungen, also fortlaufende Verhandlungen darüber, wie der Begriff Satire definiert werden kann, treten somit auf. Auch zeigen diese Beschwerden über schlechten Geschmack auf, wie Kommentierende als neues Publikum (siehe 3.1) konzeptualisiert werden können: Im Gegensatz zu anderen Satireformaten besteht bei den Memes die Möglichkeit, als Leserin der Öffentlichkeit kundzutun, dass man einen Witz nicht lustig findet. Dies lässt sich bei den Fernsehsendungen von Heute Show und Extra 3 in diesem Ausmass nicht finden. Das während der Sendungen anwesende Publikum hat nur begrenzte Möglichkeiten, seine Unzufriedenheit mit einem Scherz verbal auszudrücken.

Die dritte und grösste Gruppe sind die neutralen Kommentare, welche zu den Satiresendungen oder den Memes keine Ansichten zeigen, politisch aber vielfach klar Stellung beziehen. $\mathrm{Zu}$ dieser Gruppe gehören einerseits diejenigen, welche die Satire des Memes weiterführen und andererseits diejenigen, welche die Satire ignorieren und ernsthafte politische Argumente über die Themen ausführen, welche im Meme präsentiert werden. Die erste Gruppe besteht 
aus Kommentaren, welche sich auf die Satire im Meme beziehen, indem sie inhaltlich wie formal an diese anschliessen. Ein Beispiel hierfür ist etwa folgender Kommentar, welcher in der Kommentarsektion zum abgebildeten Meme zu finden ist:
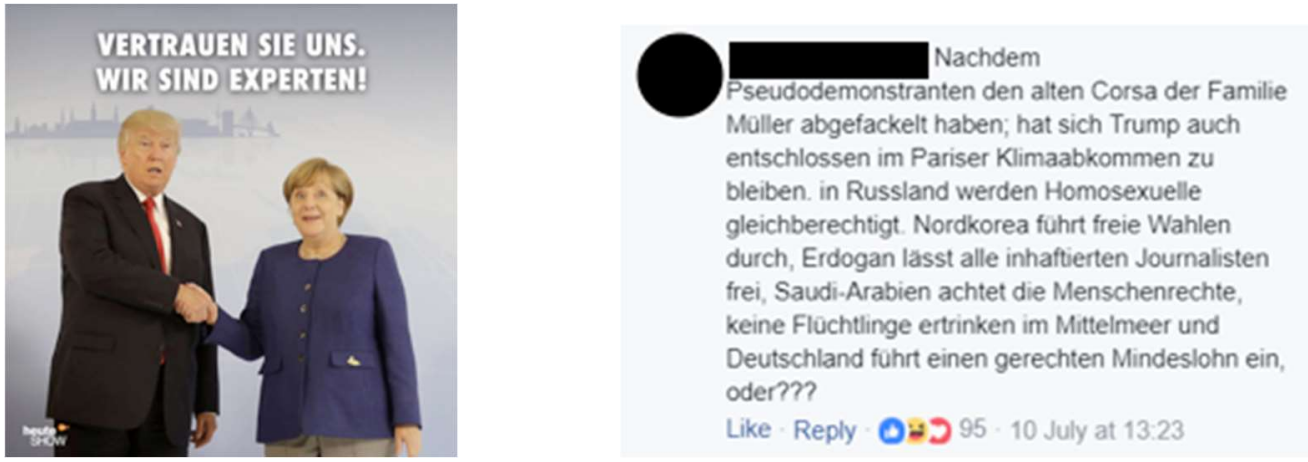

Abbildungen 21 und 22: „Wir sind Experten“-Meme (21) und dazugehöriger Kommentar

Die ironische Aussage, dass Anführer grosser Weltmächte aufgrund von Vandalismus in Hamburg ihre Politik zum Bessern ändern, wird in mehreren Memes thematisiert. Aber nicht nur in den Memes, auch in den Kommentaren zeigen sich derartige satirische Aussagen. Dies ist wiederum ein Hinweis darauf, dass sich die Kommentierenden auch als Produzenten betätigen.

Die Mehrheit der neutralen Kommentare bezieht sich jedoch nicht auf die in den Memes enthaltene Satire, sondern führt vielmehr die im Meme angesprochenen politischen Themen weiter. So löst etwa ein satirischer Kommentar zu illegal aufgestellten Demonstrationszelten eine nicht-satirische Diskussion darüber aus, ob Demonstrierende illegal in Hamburg übernachten dürfen:

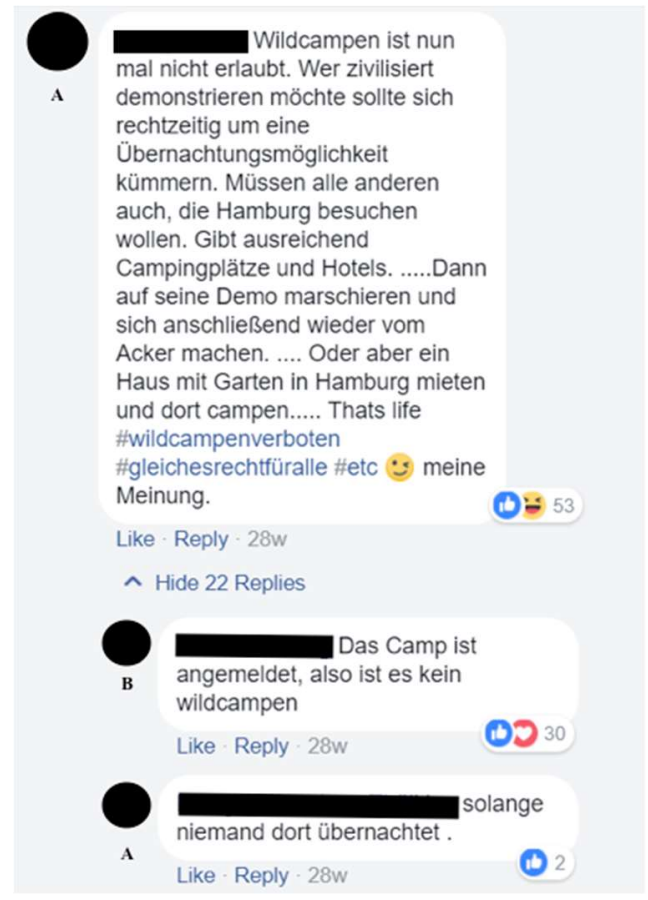

Abbildung 23: Kommentare unter „Kein Zelt ist illegal“-Meme, A und B verweisen auf die Interaktionspartner 
Obwohl der Inhalt durch das Zwinkersmiley am Ende als etwas abgeschwächt interpretiert werden kann, so ist doch klar, dass der Kommentar eine ernsthafte Meinung kundtut, was am Ende auch durch die Deklaration meine Meinung explizit markiert wird. Die Rückantworten beinhalten in den wenigsten Fällen Verweise auf das Satire-Genre, sondern nehmen eine ernsthafte politische Diskussion auf. Die Kommentarsektion wird somit als eine Plattform genutzt, auf der politische Themen besprochen werden, ohne dass darauf eingegangen werden muss, dass der Ausgangspunkt für die Diskussion ein satirisches Meme ist. Auf diese Art lassen die Memes sich deutlich als ein Fragment des übergeordneten Diskurses zum G-20 Gipfel identifizieren. Sie kommentieren diesen nicht nur satirisch, sondern geben den Anstoss zu durchaus ernsthaften Diskussionen.

Alles in allem können die in der Kommentarsektion vorhandenen Kommentare und deren Rückantworten als Beispiel für das Wesen des neuen Publikums im Sinne von Rosen (2012) aufgefasst werden. Ein Publikum für die Satire der beiden Sendungen ist auf Facebook sehr wohl vorhanden. Doch dieses Publikum mischt sich ein, führt die Satire weiter oder kritisiert gewisse Scherze als nicht lustig. Die Affordanz des Kommentierens, welche auf dieser Plattform gegeben ist, führt zu einem aktiveren Publikum, welches teilweise wiederum auch selbst zum Produzenten von Satire wird. In vielen Kommentaren finden sich auch in einer Art Introspektion metasprachliche Äusserungen über die Kommentarsektion. Einige Kommentare bemängeln das geistige Niveau in der Kommentarsektion. Andere Kommentierende behaupten, die Kommentare zur Unterhaltung zu lesen, was etwa durch folgenden, auch ausserhalb meines Datensets angewandten Meme, ausgedrückt wird:

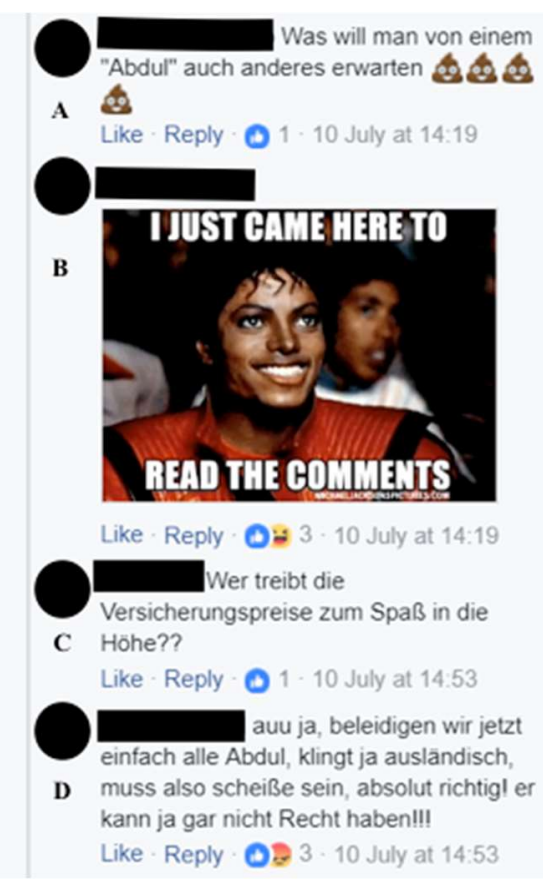

Abbildung 24: Ein Ausschnitt aus der Kommentarsektion neben dem „Wenn deine Gäste zum Hafengeburtstag“-Meme. Die Buchstaben weisen auf die unterschiedlichen Gesprächsteilnehmer hin

Das in Abbildung 24 gezeigte Meme gehört zu einem auch ausserhalb meiner Datensammlung sehr verbreiteten Popcorn-Meme. Popcorn-Memes zeigen Figuren aus der Popkultur, welche sich in Rahmen einer Vorstellung an Popcorn gütlich tun, wie in diesem Beispiel Michael Jackson im Musikvideo zu Song Thriller auf Youtube (official video). Diese Memes 
werden oft dann eingesetzt, wenn in den Kommentaren eine kontroverse Diskussion stattfindet, wo sehr viele Kommentierende aktiv sind. Das Meme drückt die Unterhaltung aus, welche der Poster beim Lesen der Kommentare empfindet. Die Unterhaltung wird mit der von abgebildeten Figuren dargestellten Emotion gleichgesetzt. Obwohl das Posten solcher Memes von Ironie begleitet ist, zeigen sie doch, welche Bedeutung die Kommentarsektion für die Lesenden haben kann: Die Kommentare werden als Spektakel konzeptualisiert, welche ebenso zur Unterhaltung beitragen, wie die von den Satiresendungen produzierten Memes.

\subsection{Diskurs - Memes - Macht}

Memes haben eine enge Bindung zum Diskurs, der in ihrem Inhalt thematisiert wird. Ohne Vorwissen über den Diskurs lässt sich der Humor und die Bedeutung der Memes nicht entziffern. Grundlingh (2018) und Osterroth (2019) betiteln dies als Kontextwissen, welches grundlegend für die Interpretierbarkeit der Memes ist. Kontext ist in der Tat von entscheidender Bedeutung, wenn es um das Verständnis geht. Im Fall von politischer Satire besteht dieser Kontext aus dem politischen Diskurs zum G-20 Gipfel. In diesem Diskurs werden die Machtverhältnisse der verschiedenen am Gipfel teilnehmenden Instanzen stark thematisiert. Auch die hier untersuchten Memes stellen dabei keine Ausnahme dar. Um die Satire in den Memes zu verstehen, wird ein Wissen um die zum Ausdruck gebrachte Diskursposition vorausgesetzt. Diese Positionen werden in den Kommentarsektionen dann oft auch kritisiert und infrage gestellt.

Die prominenteste Darstellung von Macht in den Memes ist die Dichotomie zwischen den Demonstranten und den Gipfelteilnehmenden: deren Absichten werden oft als einander entgegengesetzt gezeichnet, wobei sich die Memes bei den Demonstranten zumeist nur auf die gewaltsamen Randalierer bezieht. Diese Randalierer haben zwar die Macht, die Stadt Hamburg zu dominieren, ,in Schutt und Asche“ versinken zu lassen („Merkt-ihr-selber ne“-Meme, Abbildung 6), gleichzeitig wird ihnen keine Dominanz über die politischen Machthaber, welche die Proteste erreichen sollten, zugeschrieben. Ironisierend geben Machthaber wie Erdogan und Putin in einigen Memes vor, auf die Forderungen der Proteste einzugehen. In einem Meme nehmen die Produzentinnen sogar noch klarer Stellung. ${ }^{5}$

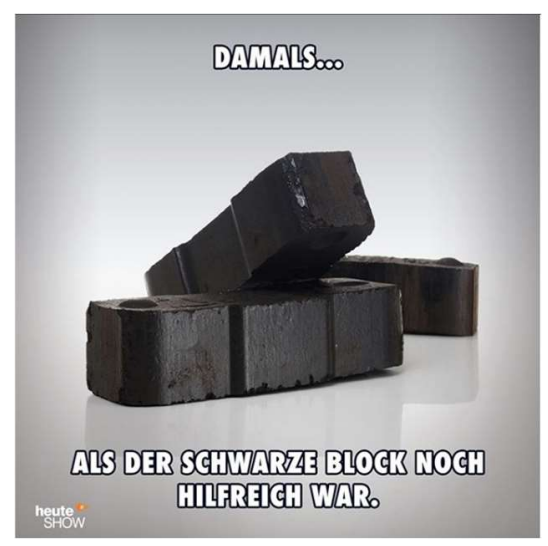

Abbildung 25: „Der Schwarze Block“-Meme

\footnotetext{
${ }^{5}$ Dieses Meme wurde aus ungeklärten Gründen nur auf Twitter und Instagram publiziert. Daher existieren in meiner Datensammlung keine Kommentare dazu.
} 
Beim Schwarzen Block handelt es sich um eine Demonstrationsstrategie schwarz gekleideter und vermummter Protestierenden, welche in Blockformation auftreten und Gewalt gegen Staatsinstitutionen und Gebäude ausdrücklich gutheissen (cf. ARD Tagesschau: „Was ist der schwarze Block“" vom 13.07.2017). Dieser schwarze Block wird hier als weniger hilfreich als ein wortwörtlicher schwarzer Kohleblock verspottet. Die Anwendung des Attributs hilfreich indiziert jedoch, dass Hilfe durchaus vonnöten wäre, auch wenn der Schwarze Block diese nicht bieten kann. Die Memes, in denen die Demonstrierenden thematisiert werden, positionieren sich nicht grundsätzlich gegen die Ideologie, welche die Demonstrierenden vertreten, sondern vielmehr gegen deren gewaltsame Methoden. Die Machthaber am G-20 Gipfel werden als dermassen distanziert von den Krawallen und Demonstrationen positioniert, dass deren tatsächliche Auseinandersetzung mit den Anliegen der Demonstrierenden an und für sich schon als humoristisch gilt.

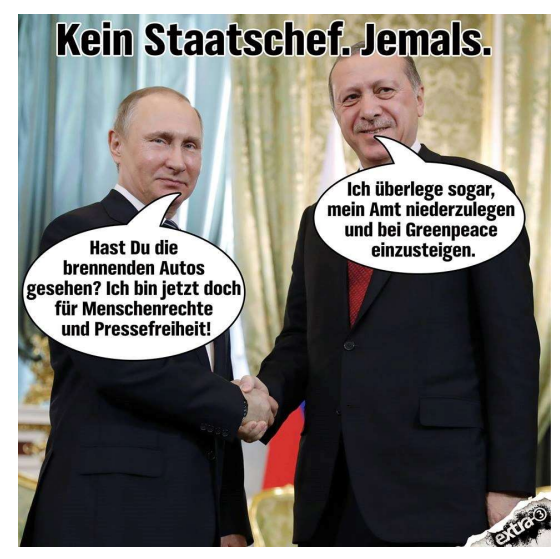

Abbildung 26: „Putin-Erdogan“-Meme

Dieses Meme stellt beispielhaft dar, wie die Machtverhältnisse in und um den G-20-Gipfel in diesem Zusammenhang diskursiv konstruiert werden. Die am Gipfel teilnehmenden Machthaber werden zwar einerseits verspottet, andererseits wird in den Memes nicht infrage gestellt, dass die Machthaber über die anderen am Gipfel teilnehmenden Instanzen dominieren. Die gewalttätigen Demonstrierenden 6 drücken durch Randale ihren Unmut mit der derzeitigen Weltordnung aus; als Beispiele werden in mehreren Memes Umweltschutz, Menschenrechte oder Pressefreiheit genannt. Im Putin-Erdogan-Meme (Abbildung 26), wo sich die beiden Machthaber ironisch mit diesen Anliegen auseinandersetzen, wird deutlich dargestellt, dass diese Randale bei den Adressaten kein ernsthaftes Gehör finden. Die Sprechblase, in welcher Putin in den Mund gelegt wird, er sei dank der gewaltsamen Proteste nun doch für Menschenrechte und Pressefreiheit, ist deshalb humoristisch, weil Putin in diesem Diskurs als dermassen autokratisch bekannt ist, dass diese Aussage nur ironisch gemeint sein kann. Die Überschrift „Kein Staatschef. Jemals.“ bestätigt diese Lesart ebenfalls. Insbesondere in den Memes von Extra 3 werden die Randale zum G-20 Gipfel als vergeblicher Aufwand angesehen, welcher die derzeitigen Machtverhältnisse eher verhärtet als auflöst. Die Hamburger Polizei und Justiz werden in den Memes ebenfalls als repressive Gewalt konstruiert, welche Demonstrierenden mit unnötiger Gewalt begegnet und deren rechtliche Handhabung sich kaum von der

\footnotetext{
${ }^{6}$ Die friedlich an Protestmärschen teilnehmenden Menschen bleiben in den Memes überwiegend unerwähnt.
} 
in autoritären Staaten unterscheidet, wie etwa untenstehendes Beispiel, welches die Form eines Quiz annimmt, zeigt:

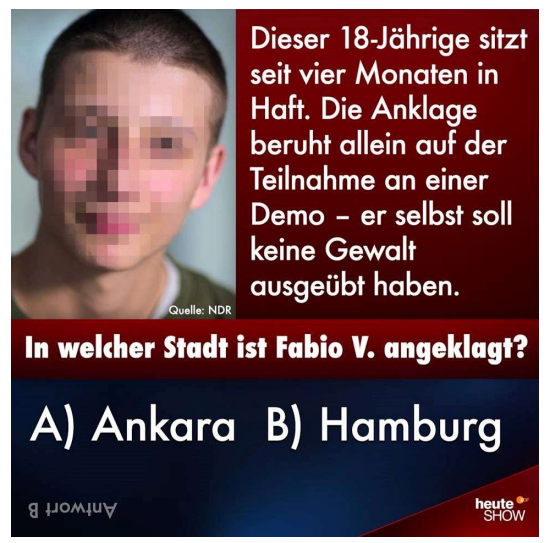

Abbildung 27: „Dieser 18-Jährige“--Meme

Im hier untersuchten Fall gehören die verschiedenen Meinungen, welche von den involvierten Instanzen vertreten werden, zum Diskurswissen: Damit sind nicht nur die Meinungen der am Gipfel beteiligten Entitäten gemeint, sondern auch die von der Heute Show und Extra 3 vertretenen Ansichten. Zum Verständnis des Memes gehört daher ein Wissen darüber, in welche Machtverhältnisse die Sendungen die am Gipfel beteiligten Entitäten einteilt. Diese werden in den Kommentaren verhandelt. Das obige Beispiel mit der Kritik am deutschen Rechtssystem wird etwa in der Kommentarsektion auch teilweise bestritten, wie folgende Topkommentare zu selbigem Meme zeigen:
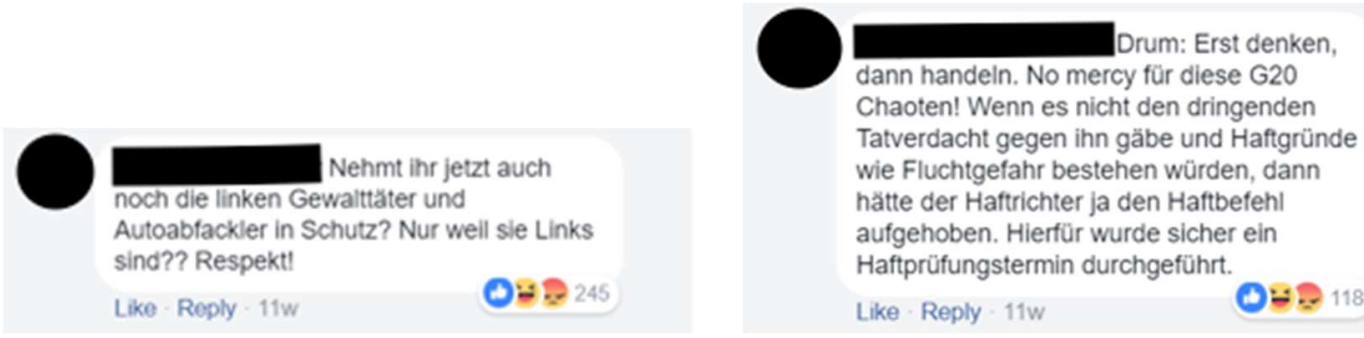

Abbildungen 28 und 29: Kommentare zu „Dieser 18-Jährige“-Meme

Den von den Sendungen präsupponierten Machtverhältnissen werden also auch widersprochen. Der fortlaufende Diskurs zu den Machverhältnissen zwischen den verschiedenen am Gipfel vertretenen Parteien zeigt sich in der Kommentarsektion neben den Memes, wo die Machtpositionen weiter verhandelt werden.

\section{$5 \quad$ Schluss}

Die hier vorliegende Studie zeigt auf, wie in den Internet Memes, ebenso wie in den dazugehörigen Fernsehsendungen, satirische Inhalte über politische Ereignisse verbreitet werden. Genau wie in den Fernsehsendungen ist auch auf Facebook ein interessiertes Publikum vorhanden, welchem die Produzentinnen durch Memes satirische Inhalte präsentieren. Satirisches Hauptwerkzeug der hier untersuchten Memes ist die Ironie. Zum Ausdruck der Ironie werden vielfach die für Memes konstitutive Kombination von Bild- und Schriftelementen benutzt. Internet Memes sind stark an das Web 2.0 gebunden und zeichnen sich durch die Nutzung gewisser Affordanzen aus, welche das Web 2.0, oder im hier untersuchten Fall die Plattform 
Facebook, seinen Nutzerinnen bietet. So lassen sich die satirischen Inhalte der Sendungen rund um die Uhr mit einem interessierten und involvierten Publikum teilen. Wichtigste Affordanz aus der Benutzerperspektive ist die Möglichkeit, die Memes in der Kommentarsektion zu kommentieren. Hiermit erhalten Kommentierende die Möglichkeit, die Satire in den Memes zu bewerten und mit anderen Kommentierenden über die im Meme angesprochenen politischen Themen zu diskutieren. Typisch für das Konzept des neuen Publikums fühlen sich die Kommentierenden hier als Mitproduzenten, halten gewisse Inhalte etwa für Verschwendung ihrer Steuergelder oder führen die Satire in ihren Kommentaren selber weiter. Dieses neue Publikum führt auch zu Herausforderungen. So lassen sich in den Kommentarsektionen vielfach Aufrufe zu Gewalt finden, gegen welche die Betreiber der Seiten trotz Netiquette kaum vorgehen. Dementsprechend werden die Diskussionen in den Kommentarsektionen oftmals hitzig geführt.

Meine Analyse zeigt also, dass satirische Texte durchaus auch im Rahmen des Webs 2.0 existieren können, auch wenn Präsentation und Rezeption sich den Gegebenheiten anpassen. Durch Memes haben Nachrichtenparodien die Möglichkeit, online an einem Diskurs teilzunehmen, der ausserhalb der Sendezeit stattfindet. Trotz der humoristischen Eigenschaft der Memes können über diese durchaus auch ernsthafte politische Botschaften satirisch vermittelt werden. In den Memes werden die Diskurspositionen der Akteure im G-20 Gipfel dargestellt, jedoch werden diese in den Kommentaren oft neu verhandelt. Wenn auch die Affordanz des Kommentierens einige Herausforderungen mit sich bringt, so ist sie doch ein wichtiger Bestandteil der Präsentation von Satire auf den Facebook-Seiten.

Die Forschungsergebnisse zeigen jedoch auch deutlich auf, dass es sich bei dieser Studie um eine erste Bestandsaufnahme eines sehr weitreichenden und komplexen Phänomens handelt, welche unbedingt durch detailliertere Forschung ergänzt werden muss. So wurde im Rahmen dieser Studie nicht genauer auf die Satirepräsentation in Memes eingegangen oder darauf, wie die Text-Bild-Kombination hier zum Zug kommt. Während in dieser Studie erste Hinweise darauf gefunden wurden, dass es sich bei den untersuchten Memes um Hybridisierungen handelt, wäre auch hier weitere Forschung vonnöten, um diese Einstufung zu bestätigen. Von Nachrichtenparodien produzierte satirische Internet Memes sind ein noch weitgehend unerforschtes Feld, wo weiterführende linguistische Forschung mit enger eingegrenzten Fragestellungen interessante Einsichten in ein gesellschaftlich relevantes Phänomen gewähren kann.

\section{Literatur- und Quellenverzeichnis}

ARD Tagesschau (2017): "Was ist der schwarze Block?" youtube.com/watch?v=3XLbleM 2rIc [28.09.20].

Bakhtin, Michail M. (1981): The Dialogic Imagination. Austin: University of Texas Press.

Baym, Geoffrey/Jones, Jeffrey (2012): „News Parody in Global Perspective: Politics, Power, and Resistance.“ In: Baym, Geoffrey/Jones, Jeffrey (eds.): News Parody in Global Perspective: Politics, Power, and Resistance. London, Routledge: 51-64.

Brummack, Jürgen (1971): „Zu Begriff und Theorie der Satire“. Deutsche Vierteljahrsschrift für Literaturwissenschaft und Geistesgeschichte. 45: 275-327.

Bülow, Lars/Merten, Marie-Luis/Johann, Michael (2018): „Internet-Memes als Zugang zu multimodalen Konstruktionen“. Zeitschrift für angewandte Linguistik. 69: 1-32. 
Castaño, Díaz/Mauricio, Carlos (2013). „Defining and Characterizing the Concept of Internet Meme.“ Revista CES Psicología 6/2: 82-104.

Caufield, Rachel P. (2012): „The Influence of ,Infoenterpropagainment': Exploring the power of political satire as a distinct form of political humor"،. In: Baumgartner, Jody/Morris, Jonathan. (eds.): Laughing Matters: Humor and American Politics in the Media Age. New York, Routledge: $3-20$.

Davison, Patrick (2012): „The Language of Internet Memes“. In: Mandiberg, Michael (ed.) The Social Media Reader. New York, New York University Press: 120-134.

Dawkins, Richard (1976): The Selfish Gene. Oxford: Oxford University Press.

Dreesen, Philipp/Kumiega, Lukasz/Spieß, Konstanze (2012): „Diskurs und Dispositiv als Gegenstände interdisziplinärer Forschung. Zur Einführung in den Sammelband“". In: Dreesen, Philipp et al.: Mediendiskursanalyse. Diskurse-Dispositive - Medien - Macht. Augsburg. Springer VS: 9-22.

Foucault, Michel (1978): Dispositive der Macht. Über Sexualität, Wissen und Wahrheit. Berlin: Merve Verlag.

Gillmor, Dan (2004): The Former Audience Joins the Party. authorama.com/we-the-media8.html [02.10.19]

Grundlingh, Lezandra (2018): „Memes as Speech Acts.“ In: Social Semiotics. Taylor and Francis/Australien: 147-168.

Hauser, Stefan/Luginbühl, Martin (2015): Hybridisierung und Ausdifferenzierung. Bern: Lang.

Heyd, Theresa (2016): Digital genres and processes of remediation. In: Georgakopoulou, Alexandra/Spilioti, Tereza (eds.): The Routledge Handbook of Language and Digital Communication. London, Routledge: 87-102.

Jackson, Michel: Thriller. Official Video. youtube.com/watch?v=sOnqjkJTMaA [08.10.19].

Jäger, Siegfried (2009): Kritische Diskursanalyse. Eine Einführung. Münster: UNRAST.

Kleinen-von Königslöw, Katharina/Kehl, Guido (2013): „Localizing The Daily Show: The Heute Show in Germany. “In: Baym, Geoffrey/Jones, Jeffrey (eds.): News Parody in Global Perspective: Politics, Power, and Resistance. London, Routledge: 51-64.

Lapp, Edgar (1992): Linguistik der Ironie. Tübingen: Narr.

Luginbühl, Martin/Perrin, Daniel (2011): „'Das, was wir in der Tagesschau den Rausschmeißer nennen': Altro- und Ethno-Kategorisierung von Textsorten im Handlungsfeld journalistischer Fernsehnachrichten“. In: Habscheid, S. (ed.): Textsorten, Handlungsmuster, Oberflächen: Linguistische Typologien der Kommunikation. Berlin/new York, De Gruyter: 577-596.

NDR: Kommentarrrichtlinien. ndr.de/service/technische_hilfe/Die-Kommentarrichtlinien, richtlinien101.html [06.03.2020].

NDR: Extra 3. ndr.de/fernsehen/sendungen/extra_3/index.html [02.10.2020].

Marx, Konstanze/Weidacher, Georg (2014): Internetlinguistik. Ein Lehr- und Arbeitsbuch. Tübingen: Narr Francke Attempto.

Marx, Konstanze (2017): Diskursphänomen Cybermobbing: Ein Internetlinguistischer Zugang zu (digitaler) Gewalt. Berlin/Boston: de Gruyter.

Milner, Ryan M. (2016): The World Made Meme. Public Conversations and Participatory Media. Cambridge: MIT Press. 
Müller, Jan-Dirk (2007): Reallexikon der deutschen Literaturwissenschaft. Bd. 3. Berlin/New Yoek: De Gruyter.

O'Reilly, Tim (2012): „What is Web 2.0? Design Patterns and Business Models for the Next Generation of Software“. In: Mandiberg, Michael (ed.): The Social Media Reader. New York, New York University Press: 32-52.

Osterroth, Andreas (2015) „Das Internet-Meme als Sprache-Bild-Text.“ IMAGE: Zeitschrift für Interdisziplinäre Bildwissenschaft 7/22: 26-46.

Osterroth, Andreas (2019) „How to do things with Memes? - Internet-Memes als multimodale Sprechakte“. In: Bülow, Lars/Johann, Michael (eds.): Politische Internet-Memes - theoretische Herausforderungen und empirische Befunde. Berlin, Frank und Timme: 41-60.

Rosen, Jay (2012): „The People formerly Known as the Audience“. In: Mandiberg, Michael (ed.) The Social Media Reader. New York, New York University Press: 13-23.

Schwarz-Friesel, Monika/Reinharz Jehuda (2013): Die Sprache der Judenfeindschaft im 21. Jahrhundert. Berlin/Boston: de Gruyter.

Schwarz-Friesel, Monika (2009) „Ironie als indirekter expressiver Sprechakt: Zur Funktion emotionsbasierter Implikaturen bei kognitiver Simulation“. In: Bachmann-Stein, Andrea/ Merten, Stephan/Roth, Christine (eds.): Perspektiven auf Wort, Satz und Text. Semantisierungsprozesse auf unterschiedlichen Ebenen des Sprachsystems. Festschrift für Inge Pohl. Trier, Wissenschaftlicher Verlag: 223-232.

Schlobinski, Peter (2005): „Editorial: Sprache und internetbasierte Kommunikation - Voraussetzungen und Perspektiven“. In: Sieves, Torsten/Schlobinski, Peter/Runkehl, Jens (eds.): Websprache.net. Sprache und Kommunikation im Internet. Berlin/New York, de Gruyter: $1-14$.

Shifman, Limor (2014): Memes in Digital Culture. Cambridge (MA): MIT Press.

Spitzmüller, Jürgen/Warnke, Ingo H. (2011): Diskurslinguistik: Eine Einführung in Theorien und Methoden der Transtextuellen Sprachanalyse. Berlin/New York: de Gruyter.

The Verge: „Facebook says it's taking new steps to stop hate speech in Sri Lanka and Myanmar". theverge.com/2019/6/21/18701073/facebook-myanmar-sri-lanka-messenger-hatespeech [30.09.2019].

Tienken, Susanne (2015): „Neue Medien, neue Formen? Hybridisierung als Aspekt des sozialen Wandels“. In: Hauser, Stefan/Luginbühl, Martin (eds.): Hybridisierung und Ausdifferenzierung. Bern etc., Lang: 31-56.

Weidacher, Georg (2019): „Sarkastische Internet-Memes im Flüchtlingsdiskurs“. In: Bülow, Lars/Johann, Michael (eds.): Politische Internet-Memes - theoretische Herausforderungen und empirische Befunde. Berlin, Frank und Timme: 41-60.

Wikipedia „kein mensch ist illegal“: de.wikipedia.org/wiki/Kein_mensch_ist_illegal [28.09.2020].

ZDF Heute Show auf Facebook: Intro. facebook.com/pg/heuteshow/about/?ref=page_internal [06.09.2019].

ZDF Heute Show auf Facebook: Netiquette. facebook.com/heuteshow/app/146387188059 1914 [06.03.2020]. 